\title{
The extremal graphs of order trees and their topological indices*
}

\author{
Rui Song ${ }^{a}$, Qiongxiang Huang ${ }^{a}{ }^{\dagger}$ and Peng Wang ${ }^{a}$ \\ ${ }^{a}$ College of Mathematics and Systems Science, Xinjiang University, Urumqi, Xinjiang 830046, China
}

\begin{abstract}
Recently, D. Vukičević and J. Sedlar in [1] introduced an order " $\leq$ " on $\mathcal{T}_{n}$, the set of trees on $n$ vertices, such that the topological index $F$ of a graph is a function defined on the order set $\left\langle\mathcal{T}_{n}, \preceq\right\rangle$. It provides a new approach to determine the extremal graphs with respect to topological index $F$. By using the method they determined the common maximum and/or minimum graphs of $\mathcal{T}_{n}$ with respect to topological indices of Wiener type and anti-Wiener type. Motivated by their researches we further study the order set $\left\langle\mathcal{T}_{n}, \leq\right\rangle$ and give a criterion to determine its order, which enable us to get the common extremal graphs in four prescribed subclasses of $\left\langle\mathcal{T}_{n}, \leq\right\rangle$. All these extremal graphs are confirmed to be the common maximum and/or minimum graphs with respect to the topological indices of Wiener type and anti-Wiener type. Additionally, we calculate the exact values of Wiener index for the extremal graphs in the order sets $\langle C(n, k), \leq\rangle$, $\left\langle\mathcal{T}_{n}(q), \leq\right\rangle$ and $\left\langle\mathcal{T}_{n}^{\Delta}, \preceq\right\rangle$.
\end{abstract}

Keywords: Order set, Tree, Extremal graph, Topological index $F$ of a graph.

AMS Classification: 05C50

\section{Introduction}

The Wiener index of a graph $G$ defined by $W(G)=\sum_{u, v \in V} d(u, v)$ is the first topological index introduced early in 1947 by H.Wiener in [2]. With time other topological indices, such as some variations and modifications of Wiener index, are introduced and studied because of their chemical applications and mathematical properties [24-27, 30]. Some of the topological indices are related with distance $d(u, v)$ as in $W(G)$. The researches for such topological indices are usually focus on some classes of graphs, especially trees, and produce many results published in various academic journals, one can refer to [20-24, 26, 27, 29, 30] for references. All theses results mainly include evaluating the bounds of the topological indices and characterizing the corresponding extremal graphs. Recently, D. Vukičević and J. Sedlar in [1] introduced an order on trees by edge division vector

\footnotetext{
*This work is supported by NSFC Grant No. 11971274, 11531011, 11671344 .

${ }^{\dagger}$ Corresponding author.

Email addresses: huangqx@xju.edu.cn(Q.X. Huang).
} 
and established the relationship between the order and topological indices. It allows us to study this problem in a uniform way and then obtain general conclusions.

Let $G$ be a simple graph on $n$ vertices. For a vertex $v \in V(G)$, let $N_{G}(v)$ be the neighbors of $v$ and $d_{G}(v)=\left|N_{G}(v)\right|$ the degree of $v$. A vertex $v$ is called a pendent vertex if $d_{G}(v)=1$. For a pair of vertices $u, v \in V(G)$ we define distance $d_{G}(u, v)$ as the length of the shortest path connecting vertices $u$ and $v$.

A tree $T$ is a connected graph without cycle. A vertex $v$ of $T$ is a branching vertex if $d_{T}(v) \geq 3$. Given a tree $T$, let $e=u v \in E(T)$ be an edge, $T_{u}$ and $T_{v}$ be respectively the two components of $T-e$ containing $u$ and $v$. By $n_{u}(e)$ (resp., $\left.n_{v}(e)\right)$ we denote the number of vertices whose distance to vertex $u$ (resp., $v$ ) is smaller than the distance to vertex $v$ (resp., $u$ ), i.e., $n_{u}(e)=\left|T_{u}\right|$ and $n_{v}(e)=\left|T_{v}\right|$. Also, we write $n_{u}^{\prime}(e)$ and $n_{v}^{\prime}(e)$ for the tree $T^{\prime}$. Furthermore, for an edge $e=u v \in E(T)$ we define $\mu(e)=\min \left\{n_{u}(e), n_{v}(e)\right\}$. By definition, we have $n_{u}(e)+n_{v}(e)=n$. It follows that $\mu(e) \leq\left\lfloor\frac{n}{2}\right\rfloor$.

Let $\mathcal{T}_{n}$ denote the set of trees on $n$ vertices. For a tree $T \in \mathcal{T}_{n}$, let $r_{i}(T)$ denote the number of edges for which $\mu(e)=i$, i.e., $r_{i}(T)=|\{e \in E(T) \mid \mu(e)=i\}|$. It is clear that $r_{1}(T)$ is just the number of pendent edges of $T$ and $r_{i}(T)=0$ for every $i>\left\lfloor\frac{n}{2}\right\rfloor$ due to $\mu(e) \leq\left\lfloor\frac{n}{2}\right\rfloor$. The edge division vector $\mathbf{r}(T)$ of a tree $T$ is defined as a vector $\mathbf{r}(T)=\left(r_{1}(T), r_{2}(T), \ldots, r_{\left\lfloor\frac{n}{2}\right\rfloor}(T)\right)$. We will write only $\mathbf{r}$ and $r_{i}$ when it does not lead to confusion. Let $\mathbf{r}$ and $\mathbf{r}^{\prime}$ be respectively the edge division vectors of $T$ and $T^{\prime}$ in $\mathcal{T}_{n}$. Recently, D. Vukičević and J. Sedlar in [1] defined an order: $\mathbf{r} \leq \mathbf{r}^{\prime}$ if and only if the inequality $\sum_{i=k}^{\left\lfloor\frac{n}{2}\right\rfloor} r_{i} \leq \sum_{i=k}^{\left\lfloor\frac{n}{2}\right\rfloor} r_{i}^{\prime}$ holds for every $k=1,2, \ldots,\left\lfloor\frac{n}{2}\right\rfloor$. If the inequality is strict for at least one $k$, then we say that $\mathbf{r} \prec \mathbf{r}^{\prime}$. Naturally, they introduced an order of trees by using edge division vector as following: for $T, T^{\prime} \in \mathcal{T}_{n}, T \leq T^{\prime}$ if $\mathbf{r} \leq \mathbf{r}^{\prime}$ (with $T<T^{\prime}$ if $\mathbf{r}<\mathbf{r}^{\prime}$ ). It is worth mentioning that such an order " $\leq$ " satisfies the reflexivity and transitivity but not antisymmetry, and so is not partial order (one can refer to the Remark 3.1), i.e., $\left\langle\mathcal{T}_{n}, \leq\right\rangle$ is not a poset but order set. Thus by $T \approx T^{\prime}$ we mean $\mathbf{r}(T)=\mathbf{r}\left(T^{\prime}\right)$. However, if a tree $T$ is uniquely determined by its edge division vector $\mathbf{r}(T)$, then $\mathbf{r}(T)=\mathbf{r}\left(T^{\prime}\right)$ implies that $T=T^{\prime}$.

Since $\mathcal{T}_{n}$ is finite set, for any subset $\mathcal{S} \subseteq \mathcal{T}_{n},\langle\mathcal{S}, \leq\rangle$ must have a unique maximum element $T^{+}$called the maximal graph (with respect to $\mathcal{S}$ ), and minimum element $T^{-}$called the minimal graph (with respect to $\mathcal{S}$ ). Also in [1] D. Vukičević and J. Sedlar established the relationship between $\left\langle\mathcal{T}_{n}, \leq\right\rangle$ and topological index $F$ of trees by defining $F: \mathcal{T}_{n} \rightarrow \mathbb{R}$ with the monotonous properties that for $T, T^{\prime} \in \mathcal{T}_{n}$, if $T \leq T^{\prime}$ implies $F(T) \leq F\left(T^{\prime}\right)$ (resp., $F(T) \geq F\left(T^{\prime}\right)$ ) then $F$ is refereed to a topological index of Wiener type (resp., anti-Wiener type). Thus if $F$ is confirmed to be a topological index of Wiener type, then $T^{+}$will be the maximum graph for this index, and $T^{-}$the minimum graph while the reverse holds for a topological index of anti-Wiener type. This gives us a general approach to find the corresponding maximum or minimum graph and evaluate bounds of topological index $F$ in some class. By using the approach, D. Vukičević and J. Sedlar in [1] proved that the $P_{n}$ and $S_{n}$ are respectively the maximal and minimal elements in $\left\langle\mathcal{T}_{n}, \preceq\right\rangle$. Moreover, by verifying the monotonicity of the topological index, they confirm that $P_{n}$ and $S_{n}$ are also the common maximum graph and/or minimum graph with respect to the indices of Wiener index, Steiner $k$-Wiener index, modified Wiener indices and variable Wiener indices, respectively.

Inspired by their research works in [1], in this paper we first give a criterion to de- 
termine the orders of trees, which leads to some graph transformations preserving this order in $\left\langle\mathcal{T}_{n}, \leq\right\rangle$, and then we determine the maximum graph and/or minimum graph in the following classes:

(1) $C(n, k)$, the set of caterpillar trees on $n$ vertices with respect to the path $P_{k}$.

(2) $\mathcal{T}_{n}(q)$, the subset of $\mathcal{T}_{n}$ with exactly $q$ pendent vertices.

(3) $\mathcal{T}(n, k-1)$, the subset of $\mathcal{T}_{n}$ with diameter $k-1$.

(4) $\mathcal{T}_{n}^{\Delta}$, the subset of $\mathcal{T}_{n}$ with maximum degree $\Delta$.

Next, by verifying the monotonicity of the topological indices, we show that the above extremal graphs are respectively the common maximum graph and/or minimum graph with respect to the following topological indices:

(1) The hyper-Wiener index proposed by M. Randić in [3], i.e.,

$$
W W(T)=\sum_{\{u, v\} \in V(T)}\left(\begin{array}{c}
1+d(u, v) \\
2
\end{array}\right)=\sum_{\{u, v\} \in V(T)}\left[\frac{1}{2} d(u, v)+\frac{1}{2} d^{2}(u, v)\right],
$$

where $d(u, v)$ is the distance between vertices $u$ and $v$.

(2) The Wiener-Hosoya index proposed by M. Randić in [4], i.e.,

$$
h(T)=\sum_{e \in E(T)}(h(e)+h[e])
$$

where $h(e)$ is the product of the numbers of the vertices in each component of $T-e$, and $h[e]$ is the product of the numbers of the vertices in each component of $T-\{u, v\}(\{u, v\}$ are two end vertices of $e$ ).

(3) The degree distance proposed by D. J. Klein et al. in [5], i.e.,

$$
D^{\prime}(T)=\sum_{\{u, v\} \in V(T)}(d(u)+d(v)) d(u, v)=4 \sum_{\{u, v\} \in V(T)} d(u, v)-n(n-1) .
$$

(4) The Gutman index proposed by I. Gutman in [6], i.e.,

$$
G u t(T)=\sum_{\{u, v\} \subseteq V(T)} d(u) d(v) d(u, v)=4 \sum_{\{u, v\} \in V(T)} d(u, v)-(2 n-1)(n-1) .
$$

(5) The second atom-bond connectivity index proposed by A. Graovac and M. Ghorbani in [7], i.e.,

$$
A B C_{2}(T)=\sum_{e=u v \in E(T)} \sqrt{\frac{n_{u}(e)+n_{v}(e)-2}{n_{u}(e) n_{v}(e)}},
$$

where $n_{u}(e)$ (resp., $\left.n_{v}(e)\right)$ denote the number of vertices whose distance to vertex $u$ (resp., $v$ ) is smaller than the distance to vertex $v$ (resp., $u$ ).

The present paper is organized as follows. In section 2, some notions and properties related with tree are introduced. In section 3, we give a lemma that is our criterion to determine the order of trees. By using this criterion we can set up some graph transformations that preserve the order in $\left\langle\mathcal{T}_{n}, \leq\right\rangle$. In section 4 , we give the maximum and minimum graphs in $\langle C(n, k), \leq\rangle$. In section 5 , we give the minimum graph in $\langle\mathcal{T}(n, k-1), \leq\rangle$. In section 6 , we give the maximum and minimum graphs in $\left\langle\mathcal{T}_{n}(q), \leq\right\rangle$. In section 7 , we give 
the maximum graph in $\left\langle\mathcal{T}_{n}^{\Delta}, \leq\right\rangle$. Finally, in section 8, we prove that all the extremal graphs mentioned above are the common maximum graph and/or minimum graph with respect to all the topological indices of $W W(T), h(T), D^{\prime}(T), G u t(T)$ and $A B C_{2}(T)$. Finally, we give a Table 3 that summarize all the related results old and new. At last of this section, as an example to determine the bounds of topological indices, we calculate the exact values of Wiener index for the extremal graphs in the order sets $\langle C(n, k), \leq\rangle,\left\langle\mathcal{T}_{n}(q), \leq\right\rangle$ and $\left\langle\mathcal{T}_{n}^{\Delta}, \leq\right\rangle$.

\section{Preliminaries}

Let $T$ be a tree of order $n$. A vertex $u$ of $T$ is said to be centroidal vertex of $T$ if $n_{u}(e) \geq \frac{n}{2}$ for any edge $e \in E(T)$ incident to $u$, and a centroidal vertex $u$ is said to be proper if $n_{u}(e)>\left\lceil\frac{n}{2}\right\rceil$ for any edge $e \in E(T)$ incident to $u$. For a centroidal vertex $u$, let $N_{T}(u)=\left\{v_{1}, v_{2}, \ldots, v_{r}\right\}$ and denote by $T_{v_{i}}\left(u v_{i}\right)$ the component of $T-u v_{i}$ containing $v_{i}$. It is clear that $u$ is a centroidal (resp., proper centroidal) vertex of $T$ if and only if $\left|T_{v_{i}}\left(u v_{i}\right)\right| \leq \frac{n}{2}$ (resp., $\left.\left|T_{v_{i}}\left(u v_{i}\right)\right|<\left\lfloor\frac{n}{2}\right\rfloor\right)$ for $i=1,2, \ldots, r$.

For a tree $T$ of order $n=2,3$, it is clear that $T$ has centroidal vertices. For $n>3$, let $v$ be a pendent vertex of $T$ adjacent to $v^{\prime}$, and then $T^{\prime}=T-v$ has centroidal vertex $u$ by induction hypothesis. Let $N_{T^{\prime}}(u)=\left\{v_{1}, v_{2}, \ldots, v_{r}\right\}$ and $T_{v_{i}}^{\prime}\left(u v_{i}\right)$ be the component of $T^{\prime}-u v_{i}$ containing $v_{i}$. By assumption, for each $1 \leq i \leq r$ we have

$$
\left|T_{v_{i}}^{\prime}\left(u v_{i}\right)\right|=n_{v_{i}}^{\prime}\left(u v_{i}\right)=n-1-n_{u}^{\prime}\left(u v_{i}\right) \leq n-1-\frac{n-1}{2}=\frac{n-1}{2}<\frac{n}{2} .
$$

On the other aspect, without loss of generality, assume that $v^{\prime}$ belongs to $T_{v_{1}}^{\prime}\left(u v_{1}\right)$. Then $T_{v_{1}}\left(u v_{1}\right)=T_{v_{1}}^{\prime}\left(u v_{1}\right)+v^{\prime} v$ and $T_{v_{i}}\left(u v_{i}\right)=T_{v_{i}}^{\prime}\left(u v_{i}\right)$ are also the components of $T-u$ containing $v_{i}$ for $i=2,3, \ldots, r$. If $\left|T_{v_{1}}^{\prime}\left(u v_{1}\right)\right|<\left\lfloor\frac{n}{2}\right\rfloor$, then $\left|T_{v_{i}}\left(u v_{i}\right)\right| \leq\left\lfloor\frac{n}{2}\right\rfloor$ for all $i=1,2, \ldots, r$, and thus $u$ is also a centroidal vertex of $T$. Otherwise, $\left|T_{v_{1}}^{\prime}\left(u v_{1}\right)\right|=\left\lfloor\frac{n}{2}\right\rfloor$. We have $\left|T_{v_{1}}\left(u v_{1}\right)\right|=\left\lfloor\frac{n}{2}\right\rfloor+1$ and thus $\left|T_{v_{2}}\left(u v_{2}\right)\right|+\cdots+\left|T_{v_{r}}\left(u v_{r}\right)\right|=n-1-\left|T_{v_{1}}\left(u v_{1}\right)\right|=n-2-\left\lfloor\frac{n}{2}\right\rfloor<\left\lfloor\frac{n}{2}\right\rfloor$. It implies that by replacing $v$ with a pendent vertex of $T$ in $T_{v_{2}}\left(u v_{2}\right)$, the centroidal vertex $u$ of $T-v$ is also a centroidal vertex of $T$. Therefore, the centroidal vertex of $T$ always exists.

Suppose that $u_{1}$ and $u_{2}$ be two centroidal vertices of $T$ and $P=u_{1} \cdots u_{2}$ be the path connecting $u_{1}$ and $u_{2}$. We claim that $P=u_{1} u_{2}$. Since otherwise, let $P=u_{1} x_{1} \cdots x_{2} u_{2}$ where $x_{1}$ may be equal to $x_{2}$. We have $n \leq\left\lceil\frac{n}{2}\right\rceil+\left\lceil\frac{n}{2}\right\rceil \leq\left|T_{u_{1}}\left(u_{1} x_{1}\right)\right|+\left|T_{u_{2}}\left(u_{2} x_{2}\right)\right| \leq n-1$, a contradiction. It also implies that $T$ has at most two centroidal vertices. Summering above arguments, we get the following simple results mentioned in [10,11].

Lemma 2.1. Let $T$ be a tree. Then the following statements hold:

(1) $T$ has at least one and at most two centroidal vertices;

(2) $T$ has two centroidal vertices $u$ and $v$ if and only if $u v$ is an edge of $T$ such that the two components of $T-u v$ have the same order.

For a tree $T$ on $n$ vertices, we say an edge $e=u v \in E(T)$ is center edge if $\mu(e)=$ $\min \left\{n_{u}(e), n_{v}(e)\right\}=\left\lfloor\frac{n}{2}\right\rfloor$. Obviously, $S_{n}$, the star on $n$ vertices, has no any center edge if $n \geq 4$. For a center edge $e=u v$, without loss of generality, we assume that $n_{u}(e) \leq n_{v}(e)$. Then $\left\lfloor\frac{n}{2}\right\rfloor=n_{u}(e) \leq n_{v}(e)=\left\lceil\frac{n}{2}\right\rceil$ since $n_{u}(e)+n_{v}(e)=n$. To exactly, we have

$$
\begin{cases}n_{u}(e)=\frac{n}{2}=n_{v}(e), & \text { if } n \text { is even } \\ \left\lfloor\frac{n}{2}\right\rfloor=n_{u}(e)<n_{v}(e)=\frac{n+1}{2}=\left\lceil\frac{n}{2}\right\rceil, & \text { if } n \text { is odd }\end{cases}
$$


It implies the following simple result.

Lemma 2.2. Let $T$ be a tree on $n$ vertices. Then the following statements hold:

(1) If $T$ has center edge $e=u v$ and $n$ is even, then $n_{u}(e)=n_{v}(e)=\frac{n}{2}$ and $u v$ is unique.

(2) If $T$ has center edge $e=u v$ and $n$ is odd, then $\left\lfloor\frac{n}{2}\right\rfloor=n_{u}(e)<n_{v}(e)=\left\lceil\frac{n}{2}\right\rceil$.

(3) $T$ has two center edges $e_{1}$ and $e_{2}$ if and only if they are adjacent with common vertex $z=e_{1} \cap e_{2}$ such that $n_{z}\left(e_{1}\right)=n_{z}\left(e_{2}\right)=\left\lceil\frac{n}{2}\right\rceil$.

(4) $T$ has at most two center edges.

Proof. (1) and (2) follows immediately from Eq. (1).

First we prove (3). On the contrary suppose that $e_{1}=u_{1} u_{2}$ and $e_{2}=v_{1} v_{2}$ are center edges not adjacent. From (1) and (2), we know that $n$ is odd and may assume that

$$
\left\{\begin{array}{l}
\left\lfloor\frac{n}{2}\right\rfloor=n_{u_{1}}\left(e_{1}\right)<n_{u_{2}}\left(e_{1}\right)=\left\lceil\frac{n}{2}\right\rceil, \\
\left\lfloor\frac{n}{2}\right\rfloor=n_{v_{1}}\left(e_{2}\right)<n_{v_{2}}\left(e_{2}\right)=\left\lceil\frac{n}{2}\right\rceil .
\end{array}\right.
$$

It implies that $u_{2}=v_{2}$. Thus $e_{1}$ and $e_{2}$ are adjacent with common vertex $u_{2}=v_{2}=z$, consequently $n_{z}\left(e_{1}\right)=n_{z}\left(e_{2}\right)=\left\lceil\frac{n}{2}\right\rceil$. Conversely, suppose that $e_{1}$ and $e_{2}$ have common vertex $z=e_{1} \cap e_{2}$ such that $n_{z}\left(e_{1}\right)=n_{z}\left(e_{2}\right)=\left\lceil\frac{n}{2}\right\rceil$. Let $e_{1}=u z$. We have $n_{u}\left(e_{1}\right)=\left\lfloor\frac{n}{2}\right\rfloor$ and so $\mu_{T}\left(e_{1}\right)=\min \left\{n_{u}\left(e_{1}\right), n_{z}\left(e_{1}\right)\right\}=\left\lfloor\frac{n}{2}\right\rfloor$. Similarly, $e_{2}$ is also a center edge with $\mu_{T}\left(e_{2}\right)=\left\lfloor\frac{n}{2}\right\rfloor$.

One can simply verify (4) from (3).

The following lemma gives some relations between centroidal vertex and center edge.

Lemma 2.3. Let $T$ be a tree on $n$ vertices. Then the following statements hold:

(1) If $T$ has two centroidal vertices $u$ and $v$, then $u v$ is the only center edge of $T$;

(2) If T has only one proper centroidal vertex if and only if T has no center edge;

(3) If $T$ has only one centroidal vertex $u$ such that $n_{u}(e)=\left\lceil\frac{n}{2}\right\rceil$ for any edge e incident to $u$ if and only if $d(u)=2$ and $T$ has two center edges incident to $u$;

(4) If $T$ has only one centroidal vertex $u$ such that $n_{u}(e)=\left\lceil\frac{n}{2}\right\rceil$ for only one edge e incident to $u$, then e is just one center edge of $T$.

Proof. Suppose that $u$ and $v$ are two centroidal vertices of $T$. Then $u v$ is an edge of $T$ and the two components of $T-e$ have the same order by Lemma2.1(2). It follows that $u v$ is a center edge and $n$ is even. By Lemma 2.2(1), the center edge $u v$ is unique and (1) follows.

In the following proofs of (2), (3) and (4), we always assume that the centroidal vertex $u$ has neighbors $v_{1}, \ldots, v_{r}$ and $T_{i}$ is the component of $T-u$ containing $v_{i}$ for $i=1, \ldots, r$.

Now we show (2). Suppose that $T$ has one proper centroidal vertex $u$. Then $n_{u}\left(u v_{i}\right)>$ $\left\lceil\frac{n}{2}\right\rceil$, and so $u v_{i}$ is not center edge since $\mu\left(u v_{i}\right)=\min \left\{n_{u}\left(u v_{i}\right), n_{v_{i}}\left(u v_{i}\right)\right\}<\left\lfloor\frac{n}{2}\right\rfloor$. For the edge $e \in E\left(T_{i}\right)$, we have $\mu(e)<\left|T_{i}\right|=n_{v_{i}}\left(u v_{i}\right)<\left\lfloor\frac{n}{2}\right\rfloor$. Thus, $T$ has no center edge. To show the converse, we assume that $\mu(e)<\left\lfloor\frac{n}{2}\right\rfloor$ for all the edge $e \in E(T)$. For $v \in V(T), N_{T}(v)$ can be partitioned as two parts $N_{T}(v)=N^{-}(v) \cup N^{+}(v)$ such that $N^{-}(v)=\left\{x \in N_{T}(v) \mid n_{x}(x v)<\right.$ $\left.\left\lfloor\frac{n}{2}\right\rfloor\right\}$ and $N^{+}(v)=\left\{y \in N_{T}(v) \mid n_{y}(y v) \geq\left\lfloor\frac{n}{2}\right\rfloor\right\}$. Now let $v^{*}$ be a vertex such that $\left|N^{+}\left(v^{*}\right)\right|$ is as small as possible. In what follows we show that $u=v^{*}$ is a proper centroidal vertex. It is clear that $v^{*}$ is a centroidal vertex if $\left|N^{+}\left(v^{*}\right)\right|=0$. On the contrary, assume that $\left|N^{+}\left(v^{*}\right)\right| \geq$ 1. We claim that $\left|N^{+}\left(v^{*}\right)\right|=1$. Since otherwise, there are two vertices $y_{1}, y_{2} \in N^{+}\left(v^{*}\right)$. We have $n_{y_{1}}\left(y_{1} v^{*}\right), n_{y_{2}}\left(y_{2} v^{*}\right) \geq\left\lfloor\frac{n}{2}\right\rfloor$, and furthermore $n_{y_{1}}\left(y_{1} v^{*}\right), n_{y_{2}}\left(y_{2} v^{*}\right)>\left\lceil\frac{n}{2}\right\rceil$ since there is no 
any center edge. Thus $n \geq n_{y_{1}}\left(y_{1} v^{*}\right)+n_{y_{2}}\left(y_{2} v^{*}\right)+1>\left\lceil\frac{n}{2}\right\rceil+\left\lceil\frac{n}{2}\right\rceil+1 \geq n+1$, a contradiction. Now we can further assume that $y_{1}$ is the unique vertex in $N^{+}\left(v^{*}\right)$ and $v^{*}$ is the unique vertex in $N^{+}\left(y_{1}\right)$ since $T$ is finite and acyclic. Thus $n_{y_{1}}\left(v^{*} y_{1}\right) \geq\left\lfloor\frac{n}{2}\right\rfloor$ and $n_{v^{*}}\left(y_{1} v^{*}\right) \geq\left\lfloor\frac{n}{2}\right\rfloor$. It implies that $\left\{n_{y_{1}}\left(v^{*} y_{1}\right), n_{v^{*}}\left(y_{1} v^{*}\right)\right\}=\left\{\left\lfloor\frac{n}{2}\right\rfloor,\left\lceil\frac{n}{2}\right\rceil\right\}$, and so $v^{*} y_{1}$ is a center edge of $T$. It is a contradiction.

Next we show (3). Suppose that $T$ has only one centroidal vertex $u$ such that $n_{u}(e)=$ $\left\lceil\frac{n}{2}\right\rceil$ for any edge $e$ incident to $u$. We have $\left|T_{i}\right|=\left\lfloor\frac{n}{2}\right\rfloor$ for $i=1,2, \ldots, r$. It implies that $r=2$ and $d(u)=2$. Therefore, $\mu\left(u v_{i}\right)=\left|T_{i}\right|=\left\lfloor\frac{n}{2}\right\rfloor$, and $u v_{1}$ and $u v_{2}$ are the two center edges. To show the converse, let $e_{1}=u v_{1}$ and $e_{2}=u v_{2}$ be two center edges. We have $n_{u}\left(e_{1}\right)=n_{u}\left(e_{2}\right)=\left\lceil\frac{n}{2}\right\rceil$ by Lemma2.2 (3). Thus the vertex $u$ is the unique centroidal vertex.

At last we show (4). Without loss of generality, we say $n_{u}\left(u v_{1}\right)=\left\lceil\frac{n}{2}\right\rceil$ and $n_{u}\left(u v_{i}\right)>\left\lceil\frac{n}{2}\right\rceil$ for $2 \leq i \leq r$. We have $\left|T_{1}\right|=\left\lfloor\frac{n}{2}\right\rfloor$ and $\left|T_{i}\right|<\left\lfloor\frac{n}{2}\right\rfloor$ for $2 \leq i \leq r$. Since $\mu\left(u v_{1}\right)=\left|T_{1}\right|=\left\lfloor\frac{n}{2}\right\rfloor$, $u v_{1}$ is a center edge. In addition, $\mu\left(u v_{i}\right)=\left|T_{i}\right|<\left\lfloor\frac{n}{2}\right\rfloor$ for $i=2, \ldots, r$, and $\mu(e)<\left|T_{i}\right| \leq\left\lfloor\frac{n}{2}\right\rfloor$ for any edge $e \in E\left(T_{i}\right)$ where $i=1, \ldots, r$. Therefore, any edge other than $e=u v_{1}$ is not center edge of $T$.

We complete this proof.

\section{A criterion to determine the order of trees}

In this section, we will give a criterion to determine the order in $\left\langle\mathcal{T}_{n}, \preceq\right\rangle$.

For $T, T^{\prime} \in \mathcal{T}_{n}$, let $\varphi: E(T) \longrightarrow E\left(T^{\prime}\right)$ be a bijection. $T$ and $T^{\prime}$ are said to be $(\varphi, \mu)$-similar with respect to $e_{1} \in E(T)$ if $\mu_{T}(e)=\mu_{T^{\prime}}(\varphi(e))$ for any $e \neq e_{1}$.

Lemma 3.1. Suppose that $T, T^{\prime} \in \mathcal{T}_{n}$ are $(\varphi, \mu)$-similar with respect to $e_{1}$, and $\varphi\left(e_{1}\right)=e_{1}^{\prime}$. We have

(1) If $\mu_{T}\left(e_{1}\right)<\mu_{T^{\prime}}\left(e_{1}^{\prime}\right)$, then $T<T^{\prime}$;

(2) If $\mu_{T}\left(e_{1}\right)>\mu_{T^{\prime}}\left(e_{1}^{\prime}\right)$, then $T>T^{\prime}$;

(3) If $\mu_{T}\left(e_{1}\right)=\mu_{T^{\prime}}\left(e_{1}^{\prime}\right)$, then $T \approx T^{\prime}$.

Proof. Let $\mathbf{r}=\left(r_{1}, \ldots, r_{\left\lfloor\frac{n}{2}\right\rfloor}\right)$ and $\mathbf{r}^{\prime}=\left(r_{1}^{\prime}, \ldots, r_{\left\lfloor\frac{n}{2}\right\rfloor}^{\prime}\right)$ be the edge division vectors of $T$ and $T^{\prime}$, respectively. By assumption, there exists a bijection $\varphi: E(T) \longrightarrow E\left(T^{\prime}\right)$ such that $\mu_{T}(e)=\mu_{T^{\prime}}(\varphi(e))$ for any $e \neq e_{1}$.

Now we prove (1). Without loss of generality, assume that $s=\mu_{T}\left(e_{1}\right)<\mu_{T^{\prime}}\left(e_{1}^{\prime}\right)=t$. First, for $i \neq s, t$ we have

$$
\begin{aligned}
r_{i}=\left|\left\{e \in E(T) \mid \mu_{T}(e)=i\right\}\right| & =\left|\left\{e \in E\left(T \backslash e_{1}\right) \mid \mu_{T}(e)=i\right\}\right| \\
& =\left|\left\{\varphi(e) \in E\left(T^{\prime} \backslash e_{1}^{\prime}\right) \mid \mu_{T^{\prime}}(\varphi(e))=i\right\}\right| \\
& =\left|\left\{e^{\prime} \in E\left(T^{\prime}\right) \mid \mu_{T^{\prime}}\left(e^{\prime}\right)=i\right\}\right|=r_{i}^{\prime} .
\end{aligned}
$$

Next, for $i=s$ we have

$$
\begin{aligned}
r_{s} & =\left|\left\{e \in E(T) \mid \mu_{T}(e)=s\right\}\right| \\
& =\left|\left\{e \in E(T) \backslash e_{1} \mid \mu_{T}(e)=s\right\}\right|+1 \\
& =\left|\left\{\varphi(e) \in E\left(T^{\prime}\right) \backslash e_{1}^{\prime} \mid \mu_{T^{\prime}}(\varphi(e))=s\right\}\right|+1 \\
& =\left|\left\{e^{\prime} \in E\left(T^{\prime}\right) \mid \mu_{T^{\prime}}\left(e^{\prime}\right)=s\right\}\right|+1 \\
& =r_{s}^{\prime}+1 .
\end{aligned}
$$


At last, for $i=t$ we can similarly get $r_{t}^{\prime}=r_{t}+1$ by exchanging the positions of $s$ and $t$. It follows that

$$
\begin{cases}\sum_{i=l}^{\left\lfloor\frac{n}{2}\right\rfloor} r_{i}=\sum_{i=l}^{\left\lfloor\frac{n}{2}\right\rfloor} r_{i}^{\prime} & \text { for } 1 \leq l \leq s, \\ \sum_{i=l}^{\left\lfloor\frac{n}{n}\right\rfloor} r_{i}<\sum_{i=l}^{\left\lfloor\frac{2}{2}\right\rfloor} r_{i}^{\prime} & \text { for } s+1 \leq l \leq t, \\ \sum_{i=l}^{\left.\frac{2}{2}\right\rfloor} r_{i}=\sum_{i=l}^{\left\lfloor\frac{2}{2}\right\rfloor} r_{i}^{\prime} & \text { for } t+1 \leq l \leq\left\lfloor\frac{n}{2}\right\rfloor .\end{cases}
$$

Therefore, we have $\mathbf{r}<\mathbf{r}^{\prime}$, and so $T<T^{\prime}$ by definition. Thus (1) follows.

To prove (2), without loss of generality, assume that $t=\mu_{T}\left(e_{1}\right)>\mu_{T^{\prime}}\left(e_{1}^{\prime}\right)=s$. By exchanging the positions of $T$ and $T^{\prime}$, and similarly as the proof of (1) one can verify that $r_{i}=r_{i}^{\prime}$ for $i \neq s, t, r_{s}^{\prime}=r_{s}+1$ and $r_{t}=r_{t}^{\prime}+1$, which leads to $T>T^{\prime}$, and so (2) follows.

According to definitions, (3) is obvious.

We complete this proof.

Remark 3.1. Obviously, if $\mathbf{r}(T) \neq \mathbf{r}\left(T^{\prime}\right)$ then $T \neq T^{\prime}$. Conversely, when $\mathbf{r}(T)=\mathbf{r}\left(T^{\prime}\right)$, however the following example shows that $T$ does not necessarily equal $T^{\prime}$. It implies that the order " $\leq$ " defined on $\mathcal{T}_{n}$ by the edge division vector is not a partial order since the antisymmetry of this order does not hold. This gives us a method to characterize graphs they are not isomorphic, but with the same index values (we can refer to Section 8).
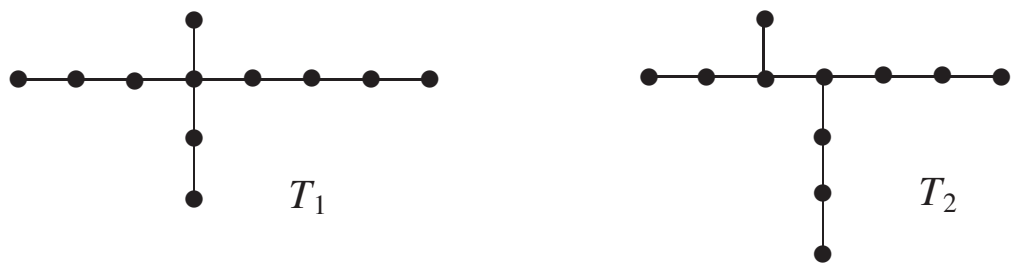

Figure 1: The graphs $T_{1}$ and $T_{2}$ with the same edge division vector $\mathbf{r}=(4,3,2,1,0)$ but $T_{1} \neq T_{2}$

For $T \in \mathcal{T}_{n}$, let $P_{k}=v_{1} \cdots v_{k}$ be a path of $T$ and $U\left(v_{i}\right)$ be the neighbors of $v_{i}$ not belong to $P_{k}$, where $1 \leq i \leq k$. For $x_{i} \in U\left(v_{i}\right)$, let $T_{x_{i}}\left(v_{i} x_{i}\right)$ be the component of $T-$ $v_{i} x_{i}$ containing $x_{i}$, where $x_{i}$ is defined to be the root vertex of the branch $T_{x_{i}}\left(v_{i} x_{i}\right)$, and $T_{v_{i}}=\cup_{x_{i} \in U\left(v_{i}\right)} T_{x_{i}}\left(v_{i} x_{i}\right)$ is a union of all such branches. Let $n_{i}=\left|T_{v_{i}}\right|=\sum_{x_{i} \in U\left(v_{i}\right)}\left|T_{x_{i}}\left(v_{i} x_{i}\right)\right|$. Obviously, $n=k+\sum_{1 \leq i \leq k} n_{i}$. Such a tree $T$ is denoted by $T\left(n ; n_{1}, \ldots, n_{k}\right)$ and called the tree with respect to path $P_{k}=v_{1} \cdots v_{k}$. All such graphs are collected in $\mathcal{T}_{n ; n_{1}, \ldots, n_{k}}$.

Let $T=T\left(n ; n_{1}, \ldots, n_{k}\right) \in \mathcal{T}_{n ; n_{1}, \ldots, n_{k}}$, where $n_{t}>0$ and $1 \leq t<k$. We call $T^{\prime}=$ $T\left(n ; n_{1}, \ldots, n_{t-1}, 0, n_{t+1}+n_{t}, \ldots, n_{k}\right)$ (see Figure 2) be the branch-shift of $T$ from $v_{t}$ to $v_{t+1}$, that can be viewed as a graph transformation from $T$ by shifting branch $T_{v_{t}}$ to $v_{t+1}$ (equivalently, deleting edges $v_{t} x_{i}$ and adding $v_{t+1} x_{i}$ for any $x_{i} \in U\left(v_{t}\right)$ ).

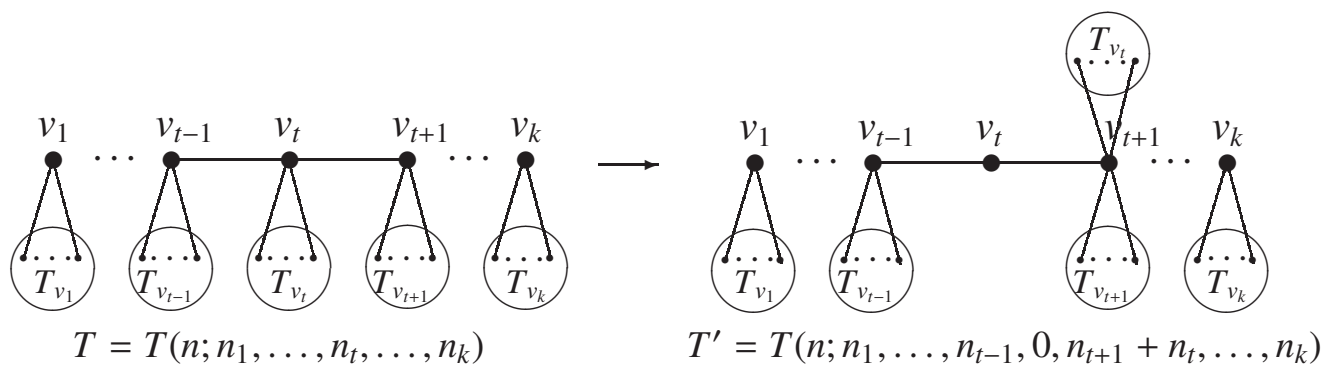

Figure 2: The branch-shift transformation 
In the following sections, we will characterize the extremal graphs with respect to the order " $\leq$ " on some prescribed families of trees. For $T=T\left(n ; n_{1}, \ldots, n_{k}\right) \in \mathcal{T}_{n ; n_{1}, \ldots, n_{k}}$ with respect to $P_{k}=v_{1} \cdots v_{k}$, we know that $T_{v_{i}}$ is a union of the branches $T_{x_{i}}\left(v_{i} x_{i}\right)$ for $x_{i} \in U\left(v_{i}\right)$. If each $T_{v_{i}}$ is an independent set then $T$ is so called the caterpillar tree which is written as $C P\left(n ; n_{1}, \ldots, n_{k}\right)$ instead of $T\left(n ; n_{1}, \ldots, n_{k}\right)$. It is clear that $\mathcal{T}_{n ; n_{1}, \ldots, n_{k}}$ contains a unique caterpillar tree $C P\left(n ; n_{1}, \ldots, n_{k}\right)$. Given $n>k$, all the caterpillar trees of the form $C P\left(n ; n_{1}, \ldots, n_{k}\right)$ satisfying $n=n_{1}+n_{2}+\cdots+n_{k}+k$ are collected in $C(n, k)$. In the next section, we will determine the extremal graphs in $\langle C(n, k), \leq\rangle$.

\section{Extremal graphs in the order set $\langle C(n, k), \leq\rangle$}

The caterpillar tree $T=C P\left(n ; n_{1}, \ldots, n_{k}\right)$ with respect to a path $P_{k}=v_{1} v_{2} \cdots v_{k}$ is a tree obtained from $P_{k}$ by attaching $n_{i}$ pendent vertices at $v_{i}$ for $i=1,2, \ldots, k$, where $n=\sum_{i=1}^{k} n_{i}+k$. In this section, we always assume that $1 \leq n_{1} \leq n_{k}$ by symmetry. Thus, if $k=n$ then $T=P_{n}$; if $k=1$ then $T=S_{n}$. Since $P_{n}$ and $S_{n}$ are unique, we may assume that $2 \leq k \leq n-1$ in what follows.

The caterpillar tree $T=C P(n ; n_{1}, \underbrace{0, \ldots, 0}_{k-2}, n_{k})$ is called the double star path and shortly for $C P_{n ; n_{1}, n_{k}}$. We call $T^{\prime}=C P_{n ; n_{1}+1, n_{k}-1}$ be the edge-shift of $T=C P_{n ; n_{1}, n_{k}}$, that can be viewed as a graph transformation from $T$ by shifting edge $v_{k} x$ to $v_{1} x$ (see Figure 3). First we give a lemma that will be used to determine the extremal graphs in $\langle C(n, k), \leq\rangle$.

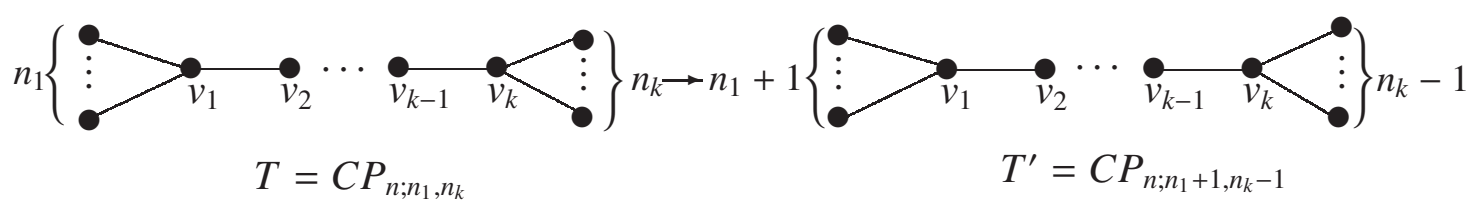

Figure 3: The edge-shift transformation

Lemma 4.1. If $n_{1}+2 \leq n_{k}$, then $T=C P_{n ; n_{1}, n_{k}} \prec T^{\prime}=C P_{n ; n_{1}+1, n_{k}-1}$.

Proof. Since $T^{\prime}$ is the edge-shift of $T$, we may assume that the pendent edge $v_{k} x_{1}$ of $T$ is shifted to $v_{1} x_{1}$. We define bijection $\varphi: E(T) \longrightarrow E\left(T^{\prime}\right)$ such that

$$
\varphi(e)= \begin{cases}e, & \text { if } e \neq v_{k} x_{1} \text { is a pendent edge of } T \\ v_{1} x_{1}, & \text { if } e=v_{k} x_{1} \\ v_{k-1} v_{k}, & \text { if } e=v_{1} v_{2} \\ v_{j-1} v_{j}, & \text { if } e=v_{j} v_{j+1} \text { where } 2 \leq j \leq k-1\end{cases}
$$

It is clear that $\mu_{T}(e)=\mu_{T^{\prime}}(\varphi(e))=1$ if $e$ is a pendent edge of $T$. In what follows, we will prove our result by distinguishing three situations to verify the condition of Lemma 3.1 .

Case 1. $T$ has no center edge.

By Lemma 2.3 (2), we see that $v_{k}$ must be the proper centroidal vertex in $T$. Thus,

$$
\left\{\begin{array}{l}
\mu_{T}\left(v_{j} v_{j+1}\right)=\min \left\{n_{1}+j, n_{k}+k-j\right\}=n_{1}+j<\left\lfloor\frac{n}{2}\right\rfloor, \\
\mu_{T^{\prime}}\left(v_{j} v_{j+1}\right)=\min \left\{n_{1}+j+1, n_{k}+k-j-1\right\}=n_{1}+j+1,
\end{array}\right.
$$


where $1 \leq j \leq k-1$. It follows that $\mu_{T}\left(v_{j} v_{j+1}\right)=\mu_{T^{\prime}}\left(v_{j-1} v_{j}\right)=\mu_{T^{\prime}}\left(\varphi\left(v_{j} v_{j+1}\right)\right)$ for $2 \leq j \leq$ $k-1$. Therefore, $T$ and $T^{\prime}$ are $(\varphi, \mu)$-similar with respect to $e_{1}=v_{1} v_{2}$, and $e_{2}=v_{k-1} v_{k}=$ $\varphi\left(e_{1}\right)$. Note that

$$
\mu_{T}\left(e_{1}\right)=\mu_{T}\left(v_{1} v_{2}\right)=n_{1}+1<n_{1}+k=\mu_{T^{\prime}}\left(v_{k-1} v_{k}\right)=\mu_{T^{\prime}}\left(e_{2}\right) .
$$

By Lemma3.1(1), we have $T<T^{\prime}$.

Case 2. $T$ has exactly one center edge.

It is clear that $n_{k}+1=\left\lceil\frac{n}{2}\right\rceil$ for $n$ is odd or $n_{k}+1 \leq \frac{n}{2}$ for $n$ is even by Lemma 2.3 (2) and (3). We may assume that $v_{h} v_{h+1}$ is the center edge of $T$, where $h \geq 3$ since $n_{1}+2 \leq n_{k}$. Thus

$$
\mu_{T}\left(v_{j} v_{j+1}\right)= \begin{cases}n_{1}+j, & \text { if } 1 \leq j \leq h \\ n_{k}+k-j, & \text { if } h+1 \leq j \leq k-1\end{cases}
$$

and

$$
\mu_{T^{\prime}}\left(v_{j} v_{j+1}\right)= \begin{cases}n_{1}+j+1, & \text { if } 1 \leq j \leq h-1 \\ n_{k}+k-j-1, & \text { if } h \leq j \leq k-1 .\end{cases}
$$

It follows that $\mu_{T}\left(v_{j} v_{j+1}\right)=\mu_{T^{\prime}}\left(v_{j-1} v_{j}\right)=\mu_{T^{\prime}}\left(\varphi\left(v_{j} v_{j+1}\right)\right)$ for $2 \leq j \leq k-1$. Therefore, $T$ and $T^{\prime}$ are $(\varphi, \mu)$-similar with respect to $e_{1}=v_{1} v_{2}$, and $e_{2}=v_{k-1} v_{k}=\varphi\left(e_{1}\right)$. Since $n_{1}+2 \leq n_{k}$ we have

$$
\mu_{T}\left(e_{1}\right)=\mu_{T}\left(v_{1} v_{2}\right)=n_{1}+1<n_{k}=\mu_{T^{\prime}}\left(v_{k-1} v_{k}\right)=\mu_{T^{\prime}}\left(e_{2}\right) .
$$

By Lemma3.1(1), we have $T<T^{\prime}$.

Case 3. $T$ has two center edges.

By Lemma2.3 (3), we see that $T$ has only one centroidal vertex and $n_{k}+1 \leq\left\lfloor\frac{n}{2}\right\rfloor$. Let $v_{h} v_{h+1}$ and $v_{h+1} v_{h+2}$ be two center edges in $T$, here $h \geq 3$ since $n_{1}+2 \leq n_{k}$. Thus, it holds that

$$
\mu_{T}\left(v_{j} v_{j+1}\right)= \begin{cases}n_{1}+j, & \text { if } 1 \leq j \leq h-1 \\ n_{1}+h=\left\lfloor\frac{n}{2}\right\rfloor, & \text { if } j=h \\ n_{k}+k-j, & \text { if } h+1 \leq j \leq k-1\end{cases}
$$

and

$$
\mu_{T^{\prime}}\left(v_{j} v_{j+1}\right)= \begin{cases}n_{1}+j+1, & \text { if } 1 \leq j \leq h-1 \\ n_{k}+k-h-1=\left\lfloor\frac{n}{2}\right\rfloor, & \text { if } j=h \\ n_{k}+k-j-1, & \text { if } h+1 \leq j \leq k-1 .\end{cases}
$$

Similarly, $T$ and $T^{\prime}$ are $(\varphi, \mu)$-similar with respect to $e_{1}=v_{1} v_{2}$, and $e_{2}=v_{k-1} v_{k}=\varphi\left(e_{1}\right)$. Since $n_{1}+2 \leq n_{k}$ we have

$$
\mu_{T}\left(e_{1}\right)=\mu_{T}\left(v_{1} v_{2}\right)=n_{1}+1<n_{k}=\mu_{T^{\prime}}\left(v_{k-1} v_{k}\right)=\mu_{T^{\prime}}\left(e_{2}\right) .
$$

By Lemma3.1(1), we have $T<T^{\prime}$.

We complete this proof.

Given $n>k \geq 2$, let $n=n_{1}+n_{k}+k$, the double star pathes $C P_{n ; n_{1}, n_{k}}$ can be ordered in the following by the Lemma 4.1.

Corollary 4.1. $C P_{n ; 0, n-k}<C P_{n ; 1, n-k-1}<C P_{n ; 2, n-k-2} \prec \cdots<C P_{n ;\left\lfloor\frac{n-k}{2}\right\rfloor,\left\lceil\frac{n-k}{2}\right\rceil}$. 


\subsection{Maximum graph in the order set $\langle C(n, k), \leq\rangle$}

In this subsection, we will show that the double star path $C P_{n ;\left\lfloor\frac{n-k}{2}\right],\left\lceil\frac{n-k}{2}\right\rceil}$ is the maximum graph in $\langle C(n, k), \leq\rangle$.

Theorem 4.1. Let $T=C P\left(n ; n_{1}, \ldots, n_{k}\right) \in C(n, k)$ be the caterpillar tree with respect to the path $P_{k}=v_{1} \cdots v_{k}$, where $2 \leq k \leq n-1$. Then $T \leq C P_{n ;\left\lfloor\frac{n-k}{2}\right\rfloor,\left\lceil\frac{n-k}{2}\right\rceil}$, with equality if and only if $T=C P_{n ;\left\lfloor\frac{n-k}{2}\right\rfloor,\left\lceil\frac{n-k}{2}\right\rceil}$.

Proof. Let $T^{*}=C P_{n ;\left\lfloor\frac{n-k}{2}\right],\left[\frac{n-k}{2}\right\rceil}, T$ be a the maximum graph in $\langle C(n, k), \preceq\rangle$ and we will show that $T=T^{*}$. By Lemma 4.1, it suffices to show that $T$ has no any branching vertex $v_{i}$ as the internal vertex of $P_{k}$. By the way of contradiction, we assume that $v_{i}$ is a branching vertex, i.e., $n_{i}>0$, for some $1<i<k$. We distinguish two situations bellow.

Case 1. $T$ has center edge.

Let $e_{h}=v_{h} v_{h+1}$ be a center edge of $T$, where $1 \leq h \leq k-1$. Then $\mu\left(e_{h}\right)=$ $\min \left\{n_{v_{h}}\left(e_{h}\right), n_{v_{h+1}}\left(e_{h}\right)\right\}=\left\lfloor\frac{n}{2}\right\rfloor$. We may assume that $n_{v_{h}}\left(e_{h}\right)=\left\lfloor\frac{n}{2}\right\rfloor$ and $n_{v_{h+1}}\left(e_{h}\right)=\left\lceil\frac{n}{2}\right\rceil$ by symmetry of $P_{k}$. Let $T^{\prime}$ be a branch-shift of $T$ from $v_{i}$ to $v_{i^{\prime}}$, where $i^{\prime}=i+1$ or $i-1$. Let $U\left(v_{i}\right)=\left\{x_{1}, \ldots, x_{n_{i}}\right\}$ be the pendent vertices sticking at $v_{i}$. Now we define a bijection $\varphi: E(T) \longrightarrow E\left(T^{\prime}\right)$ such that

$$
\varphi(e)= \begin{cases}e, & \text { if } e \neq v_{i} x_{l} \text { for any } x_{l} \in U\left(v_{i}\right) \\ v_{i^{\prime}} x_{l}, & \text { if } e=v_{i} x_{l} \text { for } x_{l} \in U\left(v_{i}\right) .\end{cases}
$$

It is clear that $\mu_{T}(e)=\mu_{T^{\prime}}(\varphi(e))$ if $e \neq v_{i} v_{i^{\prime}}$, and $\varphi\left(v_{i} v_{i^{\prime}}\right)=v_{i} v_{i^{\prime}}$. Therefore, $T$ and $T^{\prime}$ are $(\varphi, \mu)$-similar with respect to $e_{i}=v_{i} v_{i^{\prime}}$. If $i \leq h$, we can take $i^{\prime}=i-1$ and have $\mu_{T}\left(e_{i}\right)=n_{v_{i-1}}\left(e_{i}\right)<n_{v_{i-1}}\left(e_{i}\right)+n_{i}=\mu_{T^{\prime}}\left(e_{i}\right)<\left\lfloor\frac{n}{2}\right\rfloor$. By Lemma 3.1 (1), we have $T<T^{\prime}$ which contradicts the maximum hypothesis of $T$. If $i \geq h+1$, we take $i^{\prime}=i+1$. Clearly, if $i>h+1$ then $\mu_{T}\left(e_{i}\right)=n_{v_{i+1}}\left(e_{i}\right)<n_{v_{i+1}}\left(e_{i}\right)+n_{i}=\mu_{T^{\prime}}\left(e_{i}\right)<\left\lfloor\frac{n}{2}\right\rfloor$; if $i=h+1$, then $n_{v_{h+2}}\left(e_{h+1}\right)=n_{v_{h+2}}\left(v_{h+1} v_{h+2}\right)=n_{v_{h+1}}\left(e_{h}\right)-1-n_{h+1}=\left\lceil\frac{n}{2}\right\rceil-1-n_{h+1}$, and we also have $\mu_{T}\left(e_{h+1}\right)=n_{v_{h+2}}\left(e_{h+1}\right)<n_{v_{h+2}}\left(e_{h+1}\right)+n_{h+1}=\mu_{T^{\prime}}\left(e_{h+1}\right) \leq\left\lfloor\frac{n}{2}\right\rfloor$. Thus $T<T^{\prime}$ by Lemma 3.1 (1), a contradiction.

Case 2. $T$ has no center edge.

According to Lemma2.3 (2), $T$ has only one proper centroidal vertex $v_{h}$, where $1 \leq$ $h \leq k$. Then $n_{v_{h+1}}\left(v_{h} v_{h+1}\right), n_{v_{h-1}}\left(v_{h-1} v_{h}\right)<\left\lfloor\frac{n}{2}\right\rfloor$. We also consider $T^{\prime}$ defined in Case 1 . If $i<h$, we can take $i^{\prime}=i-1$ and have $\mu_{T}\left(e_{i}\right)=n_{v_{i-1}}\left(e_{i}\right)<n_{v_{i-1}}\left(e_{i}\right)+n_{i}=\mu_{T^{\prime}}\left(e_{i}\right)<\left\lfloor\frac{n}{2}\right\rfloor$; if $i>h$, we can take $i^{\prime}=i+1$ and have $\mu_{T}\left(e_{i}\right)=n_{v_{i+1}}\left(e_{i}\right)<n_{v_{i+1}}\left(e_{i}\right)+n_{i}=\mu_{T^{\prime}}\left(e_{i}\right)<\left\lfloor\frac{n}{2}\right\rfloor$. It follows that $T \prec T^{\prime}$ as in Case 1. If $i=h$, we can take $T^{\prime \prime}$ that is obtained from $T$ by shifting exactly one edge $v_{h} x_{1}$ to $v_{h-1} x_{1}$, and define

$$
\psi(e)= \begin{cases}e, & \text { if } e \neq v_{h} x_{1} \\ v_{h-1} x_{1}, & \text { if } e=v_{h} x_{1} .\end{cases}
$$

Clearly, $T$ and $T^{\prime \prime}$ are $(\psi, \mu)$-similar with respect to $e_{h-1}=v_{h-1} v_{h}$. We have $\mu_{T}\left(e_{h-1}\right)=$ $n_{v_{h-1}}\left(e_{h-1}\right)<n_{v_{h-1}}\left(e_{h-1}\right)+1=\mu_{T^{\prime \prime}}\left(e_{h-1}\right) \leq\left\lfloor\frac{n}{2}\right\rfloor$. By Lemma 3.1 (1), we have $T<T^{\prime \prime}$, a contradiction.

We complete this proof. 


\subsection{Minimum graph in the order set $\langle C(n, k), \leq\rangle$}

For $1 \leq s \leq k$, let $C P_{n, k}^{s}=C P(n ; \underbrace{0, \ldots, 0}_{s-1}, n-k, \underbrace{0, \ldots, 0}_{k-s})$ denote the caterpillar tree shown in Figure 4. We always assume that $s \leq\left\lceil\frac{k}{2}\right\rceil$ because of $C P_{n, k}^{s}=C P_{n, k}^{k-s+1}$ by symmetry.

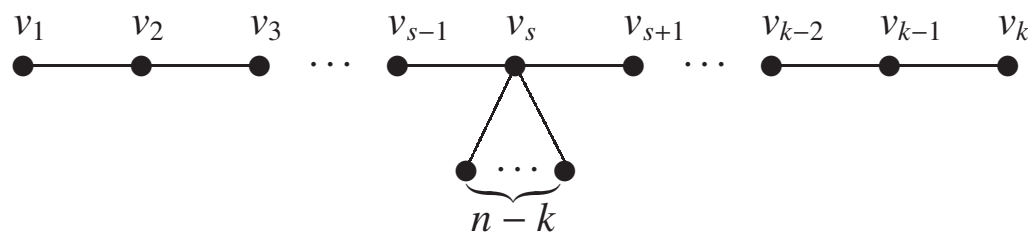

Figure 4: The graph $C P_{n, k}^{s}$

Notice that $C P_{n, 1}^{1}=C P(n ; n-1)$ and $C P_{n, 2}^{1}=C P(n ; n-2,0)$ are the star $K_{1, n-1}$, in general we always assume that $3 \leq k \leq n-1$. In this subsection, we will prove that the caterpillar tree $C P_{n, k}^{\left\lceil\frac{k}{2}\right\rceil}$ is the minimum graph in $\langle C(n, k), \leq\rangle$.

Lemma 4.2. Let $C P_{n, k}^{s}$ be the caterpillar tree with respect to $P_{k}=v_{1} v_{2} \cdots v_{k}$, where $k \geq 3$, $s<\frac{k}{2}$ and $n-k \geq 1$, then $C P_{n, k}^{s}>C P_{n, k}^{s+1}$.

Proof. Let $T=C P_{n, k}^{s}$, and $T^{\prime}=C P_{n, k}^{s+1}$ is just obtained from $T$ by shifting branch $T_{v_{s}}$ from $v_{s}$ to $v_{s+1}$. Let $U\left(v_{s}\right)=\left\{x_{1}, \ldots, x_{n-k}\right\}$ be the pendent vertices sticking at $v_{s}$. Now we define a bijection $\varphi: E(T) \longrightarrow E\left(T^{\prime}\right)$ such that

$$
\varphi(e)= \begin{cases}e, & \text { if } e \neq v_{s} x_{i} \text { for any } x_{i} \in U\left(v_{s}\right) \\ v_{s+1} x_{i}, & \text { if } e=v_{s} x_{i} \text { for } x_{i} \in U\left(v_{s}\right)\end{cases}
$$

We know that $T$ and $T^{\prime}$ are $(\varphi, \mu)$-similar with respect to $e_{s}=v_{s} v_{s+1}$, and $e_{s}=\varphi\left(e_{s}\right)$. Also note that $s<\frac{k}{2}$ and $n-k \geq 1$, for the edge $e_{s}=v_{s} v_{s+1}$ we have

$$
\begin{aligned}
\mu_{T}\left(e_{s}\right) & =\min \{s+n-k, k-s\} \\
& >s \\
& =\min \{s, n-s\} \\
& =\mu_{T^{\prime}}\left(e_{s}\right) .
\end{aligned}
$$

It follows $C P_{n, k}^{s}>C P_{n, k}^{s+1}$ by the Lemma 3.1(2).

Theorem 4.2. Let $T=C P\left(n ; n_{1}, \ldots, n_{k}\right) \in C(n, k)$ be the caterpillar tree respect to the path $P_{k}=v_{1} \cdots v_{k}$, where $2 \leq k \leq n-1$. Then $T \geq C P_{n, k}^{\left\lceil\frac{k}{2}\right\rceil}$, with equality if and only if $T=C P_{n, k}^{\left\lceil\frac{k}{2}\right\rceil}$.

Proof. If $k=2$, then $T=C P\left(n ; n_{1}, n_{2}\right)$. Since $C P(n ; 0, n-2)=C P_{n, 2}^{1}$ is also a star $S_{n}$, we have $C P\left(n ; n_{1}, n_{2}\right) \geq C P(n ; 0, n-2)=C P_{n, 2}^{1}=S_{n}$ by the Corollary 4.1. In what follows, we assume that $k \geq 3$ and distinguish three cases with regard to the number of center edges in $T$.

Let $T^{*}=C P_{n, k}^{\left\lceil\frac{k}{2}\right\rceil}, T$ be a minimum graph in $\langle C(n, k), \leq\rangle$ and we will show that $T=T^{*}$. By Lemma 4.2, it suffices to show that $T$ has exactly one branching vertex $v_{i}$ as the internal 
vertex of $P_{k}$. By the way of contradiction, we assume that $v_{l}$ and $v_{t}$ are branching vertices, i.e., $n_{l}, n_{t}>0$, for some $1 \leq l<t \leq k$. We distinguish two situations bellow.

Case 1. $T$ has a center edge.

Let $e_{h}=v_{h} v_{h+1}$ be a center edge of $T$, where $1 \leq h \leq k-1$. Then $\mu\left(e_{h}\right)=$ $\min \left\{n_{v_{h}}\left(e_{h}\right), n_{v_{h+1}}\left(e_{h}\right)\right\}=\left\lfloor\frac{n}{2}\right\rfloor$. We may assume that $n_{v_{h}}\left(e_{h}\right)=\left\lfloor\frac{n}{2}\right\rfloor$ and $n_{v_{h+1}}\left(e_{h}\right)=\left\lceil\frac{n}{2}\right\rceil$ by symmetry of $P_{k}$. First assume that $l=h$ and $t=h+1$. Let $T^{\prime}$ be a branch-shift of $T$ from $v_{h}$ to $v_{h+1}$. Let $U\left(v_{h}\right)=\left\{x_{1}, \ldots, x_{n_{h}}\right\}$ be the pendent vertices sticking at $v_{h}$. Now we define a bijection $\varphi: E(T) \longrightarrow E\left(T^{\prime}\right)$ such that

$$
\varphi(e)= \begin{cases}e, & \text { if } e \neq v_{h} x_{i} \text { for any } x_{i} \in U\left(v_{h}\right) \\ v_{h+1} x_{i}, & \text { if } e=v_{h} x_{i} \text { for } x_{i} \in U\left(v_{h}\right) .\end{cases}
$$

It is clear that $\mu_{T}(e)=\mu_{T^{\prime}}(\varphi(e))$ if $e \neq v_{h} v_{h+1}$, and $\varphi\left(v_{h} v_{h+1}\right)=v_{h} v_{h+1}$. Therefore, $T$ and $T^{\prime}$ are $(\varphi, \mu)$-similar with respect to $e_{h}=v_{h} v_{h+1}$. We have $\left\lfloor\frac{n}{2}\right\rfloor=\mu_{T}\left(e_{h}\right)=n_{v_{h}}\left(e_{h}\right)>$ $n_{v_{h}}\left(e_{h}\right)-n_{h}=\mu_{T^{\prime}}\left(e_{h}\right)$. By Lemma3.1(2), we have $T>T^{\prime}$ which contradicts the minimum hypothesis of $T$. Next assume that $l<h$ or $t>h+1$. Without loss of generality, let $l<h$ by symmetry of $P_{k}$. Let $T^{\prime \prime}$ be a branch-shift of $T$ from $v_{l}$ to $v_{l+1}$ and define a bijection $\psi: E(T) \longrightarrow E\left(T^{\prime \prime}\right)$ such that

$$
\psi(e)= \begin{cases}e, & \text { if } e \neq v_{l} x_{i} \text { for any } x_{i} \in U\left(v_{l}\right) \\ v_{l+1} x_{i}, & \text { if } e=v_{l} x_{i} \text { for } x_{i} \in U\left(v_{l}\right) .\end{cases}
$$

It is clear that $\mu_{T}(e)=\mu_{T^{\prime \prime}}(\psi(e))$ if $e \neq v_{l} v_{l+1}$, and $\varphi\left(v_{l} v_{l+1}\right)=v_{l} v_{l+1}$. Therefore, $T$ and $T^{\prime \prime}$ are $(\psi, \mu)$-similar with respect to $e_{l}=v_{l} v_{l+1}$. We have $\left\lfloor\frac{n}{2}\right\rfloor>\mu_{T}\left(e_{l}\right)=n_{v_{l}}\left(e_{l}\right)>n_{v_{l}}\left(e_{l}\right)-n_{l}=$ $\mu_{T^{\prime \prime}}\left(e_{l}\right)$. By Lemma 3.1(2), we have $T>T^{\prime \prime}$, a contradiction again.

Case 2. $T$ has no center edge.

According to Lemma 2.3 (2), $T$ has only one proper centroidal vertex $v_{h}$. Hence $n_{v_{h+1}}\left(v_{h} v_{h+1}\right), n_{v_{h-1}}\left(v_{h-1} v_{h}\right)<\left\lfloor\frac{n}{2}\right\rfloor$. Then at least one of $l<h$ and $t>h$ will occur. We may assume that $l<h$ by symmetry of $P_{k}$. Again let $T^{\prime \prime}$ be a branch-shift of $T$ from $v_{l}$ to $v_{l+1}$ and define $\psi$ as in Eq. (2), we have $T>T^{\prime \prime}$, a contradiction.

We complete this proof.

From Theorem 4.1 and Theorem 4.2, we obtain the following result.

Corollary 4.2. Let $T=C P\left(n ; n_{1}, \ldots, n_{k}\right) \in C(n, k)$. Then $C P_{n, k}^{\left\lceil\frac{k}{2}\right\rceil} \leq T \leq C P_{n ;\left\lfloor\frac{n-k}{2}\right\rfloor,\left\lceil\frac{n-k}{2}\right\rceil}$, where $2 \leq k \leq n-1$.

Denote by $\mathcal{T}(n, k-1)$ the set of trees of order $n$ in which each tree has diameter $k-1$. In next section we will consider the extremal graph in $\langle\mathcal{T}(n, k-1), \leq\rangle$.

\section{The minimum graph in the order set $\langle\mathcal{T}(n, k-1), \leq\rangle$}

Theorem 4.2 conforms that $C P_{n, k}^{\left\lceil\frac{k}{2}\right\rceil}$ is the minimum graph among $\langle C(n, k), \leq\rangle$, and $C P_{n, k}^{\left\lceil\frac{k}{2}\right\rceil} \in \mathcal{T}(n, k-1)$ for $k \geq 3$. To prove that $C P_{n, k}^{\left\lceil\frac{k}{2}\right\rceil}$ is also the minimum graph among $\langle\mathcal{T}(n, k-1), \leq\rangle$, we need the following edge-moving transformation. Let $T_{1}$ and $T_{2}$ be two 
trees of order $n_{1} \geq 2$ and $n_{2} \geq 2$, respectively, $T$ be the tree obtained from $T_{1}$ and $T_{2}$ by adding an edge between a vertex $u$ of $T_{1}$ and a vertex $v$ of $T_{2}$, and $T^{\prime}$ be the tree obtained from $T$ by contracting $u v$ to a vertex $u$ and attaching a pendent vertex $v$ to $u$ (shown in Figure 5). We call $T^{\prime}$ be the edge-moving transformation of $T$ with respect to $u v$.
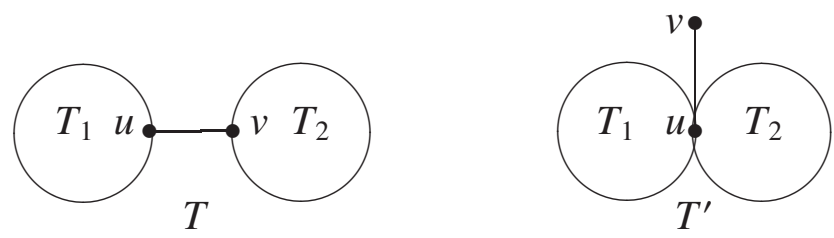

Figure 5: The graphs $T$ and $T^{\prime}$

Lemma 5.1. Let $T$ be a tree with order $n \geq 4$. If $T^{\prime}$ is the edge-moving transformation of $T$ with respect to $u v$, then $T>T^{\prime}$.

Proof. We define $\varphi: E(T) \longrightarrow E\left(T^{\prime}\right)$ such that $\varphi(e)=e$ for any edge $e \in E(T)$. It is clear that $\mu_{T}(e)=\mu_{T^{\prime}}(\varphi(e))$ if $e \neq u v$. Therefore, $T$ and $T^{\prime}$ are $(\varphi, \mu)$-similar with respect to $e_{1}=u v$.

$$
\mu_{T}\left(e_{1}\right)=\mu_{T}(u v)=\min \left\{\left|T_{1}\right|,\left|T_{2}\right|\right\}>1=\mu_{T^{\prime}}(u v)=\mu_{T^{\prime}}\left(e_{1}\right) .
$$

It follows $T>T^{\prime}$ by Lemma 3.1(2).

For $T \in \mathcal{T}(n, k-1), T$ can be presented by $T=T\left(n ; 0, n_{2}, \ldots, n_{k-1}, 0\right) \in \mathcal{T}_{n ; 0, n_{2}, \ldots, n_{k-1}, 0}$, i.e., $T=T\left(n ; 0, n_{2}, \ldots, n_{k-1}, 0\right)$ is a tree with respect to $P_{k}=v_{1} v_{2} \cdots v_{k}$ such that $\left|T_{v_{i}}\right|=n_{i}$ for $i=2,3, \ldots, k-1$. By edge-moving transformation, we can transfer the edges of $T_{v_{i}}$, one by one, to pendent edges sticking at $v_{i}$, and finally we get the caterpillar tree $C P\left(n ; 0, n_{2}, \ldots, n_{k-1}, 0\right)$ from $T\left(n ; 0, n_{2}, \ldots, n_{k-1}, 0\right)$. Therefore, by applying Lemma 5.1, we have $C P\left(n ; 0, n_{2}, \ldots, n_{k-1}, 0\right) \leq T\left(n ; 0, n_{2}, \ldots, n_{k-1}, 0\right)$. By applying Theorem 4.2, we have $C P_{n, k}^{\left\lceil\frac{k}{2}\right\rceil} \leq C P\left(n ; 0, n_{2}, \ldots, n_{k-1}, 0\right)$, and the equality holds if and only if $T=C P_{n, k}^{\left\lceil\frac{k}{2}\right\rceil}$. It follows the result.

Theorem 5.1. For $T \in \mathcal{T}(n, k-1), T \geq C P_{n, k}^{\left\lceil\frac{k}{2}\right\rceil}$ with equality holds if and only if $T=C P_{n, k}^{\left\lceil\frac{k}{2}\right\rceil}$.

By repeating applying Lemma 5.1, we get the order of minimum graphs with different diameters but the same order $n$ as follows:

Corollary 5.1. $S_{n}=C P_{n, 2}^{1}=C P_{n, 3}^{2} \prec C P_{n, 4}^{2} \prec \cdots \prec C P_{n, n-2}^{\left\lceil\frac{n-2}{2}\right\rceil} \prec C P_{n, n-1}^{\left\lceil\frac{n-1}{2}\right\rceil} \prec P_{n}$.

By Theorem 4.1 and Corollary 5.1, we have the known result in [1].

Corollary 5.2. For $T \in \mathcal{T}_{n}$, we have $S_{n} \leq T \leq P_{n}$.

Remark 5.1. We know from Theorem 4.2 and Theorem 5.1 that $C P_{n, k}^{\left[\frac{k}{2}\right\rceil}$ is the common minimum graph in $\langle C(n, k), \leq\rangle$ and $\langle\mathcal{T}(n, k-1), \leq\rangle$, respectively. From Corollary 4.2, $C P_{n ;\left\lfloor\frac{n-k+2}{2}\right],\left[\frac{n-k+2}{2}\right\rceil}$ is the maximum graph in $\langle C(n, k-2), \leq\rangle$ with diameter $k-1$. However, the maximum graph in $\langle\mathcal{T}(n, k-1), \leq\rangle$ leaves unknown. 


\section{Extremal graphs in the order set $\left\langle\mathcal{T}_{n}(q), \leq\right\rangle$}

Let $\mathcal{T}_{n}(q)$ be a set of trees with order $n$ and $q$ pendent vertices. In this section, we will give the maximum and minimum graphs in $\left\langle\mathcal{T}_{n}(q), \leq\right\rangle$. If $q=2$, then $\mathcal{T}_{n}(2)$ contains a unique graph $P_{n}=C P_{n ; 1,1}$. Similarly $S_{n}=C P_{n ;\left\lfloor\frac{n-1}{2}\right\rfloor,\left[\frac{n-1}{2}\right\rceil}$ is a unique graph in $\mathcal{T}_{n}(n-1)$. Thus we always assume that $2<q<n-1$ in this section.

\subsection{Maximum graph in the order $\operatorname{set}\left\langle\mathcal{T}_{n}(q), \leq\right\rangle$}

Recall that $C P_{n ;\left\lfloor\frac{q}{2}\right\rfloor,\left[\frac{q}{2}\right\rceil}$ is the double star path obtained from the path $P_{n-q}=v_{1} v_{2} \cdots v_{n-q}$ by averagely sticking $q$ pendent vertices at $v_{1}$ and $v_{k}$ (see Figure 3 ), respectively. It is clear that $C P_{n ;\left\lfloor\frac{q}{2}\right\rfloor,\left\lceil\frac{q}{2}\right\rceil} \in \mathcal{T}_{n}(q)$. In this section, we will show that $C P_{n ;\left\lfloor\frac{q}{2}\right\rfloor,\left\lceil\frac{q}{2}\right\rceil}$ is the unique maximum graph in $\left\langle\mathcal{T}_{n}(q), \leq\right\rangle$.

Theorem 6.1. Let $T \in \mathcal{T}_{n}(q)$, where $2<q<n-1$. Then $T \leq C P_{n ;\left\lfloor\frac{q}{2}\right\rfloor,\left\lceil\frac{q}{2}\right\rceil}$, with equality if and only if $T=C P_{n ;\left\lfloor\frac{q}{2}\right\rfloor,\left\lceil\frac{q}{2}\right\rceil}$.

Proof. If $q=n-2$, it is clear that $T \leq C P_{n ;\left\lfloor\frac{n-2}{2}\right],\left[\frac{n-2}{2}\right\rceil}$ by Lemma 4.1, In what follows, we assume that $q \leq n-3$. Let $T$ be a maximum graph in $\left\langle\mathcal{T}_{n}(q), \leq\right\rangle$. We will show that $T=C P_{n ;\left\lfloor\frac{q}{2}\right\rfloor,\left\lceil\frac{q}{2}\right\rceil}$ in what follows. According to Lemma 2.1 (1), we may assume that $u$ is a centroidal vertex of $T$ with neighbors $v_{1}, \ldots, v_{r}$. For an edge $e_{i}=u v_{i}$, let $T_{i}=T_{v_{i}}\left(e_{i}\right)$ denote the component of $T-e_{i}$ containing $v_{i}$. Without loss of generality, let $m_{i}$ be the order of $T_{i}$ such that $1 \leq m_{1} \leq m_{2} \leq \cdots \leq m_{r}, q_{i}$ be the number of pendent vertices of $T$ in $T_{i}$ for $i=1,2, \ldots, r$, and thus $q=\sum_{i=1}^{r} q_{i}$. It means that each $T_{i}$ has $m_{i}$ vertices and contains $q_{i}$ pendent vertices of $T$. Since $q \leq n-3$, there exists $m_{t}>1$ for some $1 \leq t \leq r$. Denote by $S_{m_{t}}\left(q_{t}\right)=C P_{m_{t} ; 0, q_{t}}$ the tree consisting of a path $P_{m_{t}-q_{t}}=z_{1} \cdots z_{m_{t}-q_{t}}$ and sticking $q_{t}$ pendent vertices at $z_{m_{t}-q_{t}}$, where $z_{1}=v_{t}$.

First we show that $T_{t}=S_{m_{t}}\left(q_{t}\right)$. In fact, it suffices to prove that $T_{t}$ has at most one vertex of degree great than two in $T$. Otherwise, there exists two adjacent vertices $x_{1}$ and $x_{1}^{\prime}$ of $T_{t}$ with $d_{T}\left(x_{1}\right) \geq 3$ and $d_{T}\left(x_{1}^{\prime}\right) \geq 2$ such that $H=v_{t} \cdots x_{1} x_{1}^{\prime}$ is a path in $T_{t}$, where $x_{1}$ may be equal to $v_{t}$. Let $y_{1} \in N\left(x_{1}\right) \backslash H$, and $T_{y_{1}}\left(x_{1} y_{1}\right)$ be the component of $T-x_{1} y_{1}$ containing $y_{1}$. We construct $T^{\prime}$ obtained from $T$ by deleting edge $x_{1} y_{1}$ and adding $x_{1}^{\prime} y_{1}$. Clearly, $T^{\prime} \in \mathcal{T}_{n}(q)$. We define $\varphi: E(T) \longrightarrow E\left(T^{\prime}\right)$ such that

$$
\varphi(e)= \begin{cases}e, & \text { if } e \neq x_{1} y_{1} \text { is any edge of } T \\ x_{1}^{\prime} y_{1}, & \text { if } e=x_{1} y_{1} .\end{cases}
$$

It is clear that $\mu_{T}(e)=\mu_{T^{\prime}}(\varphi(e))$ if $e \neq x_{1} x_{1}^{\prime}$. Therefore, $T$ and $T^{\prime}$ are $(\varphi, \mu)$-similar with respect to $e_{1}=x_{1} x_{1}^{\prime}$, and $\varphi\left(e_{1}\right)=e_{1}$. Additionally, since $u$ is a centroidal vertex of $T$, we have

$$
\mu_{T^{\prime}}\left(e_{1}\right)=\mu_{T}\left(x_{1}^{\prime} x_{1}\right)+\left|T_{y_{1}}\left(x_{1} y_{1}\right)\right|>\mu_{T}\left(x_{1}^{\prime} x_{1}\right)=\mu_{T}\left(e_{1}\right) .
$$

By Lemma 3.1 (1), we have $T \prec T^{\prime}$. However, $T^{\prime} \in \mathcal{T}_{n}(q)$, this is a contradiction. It implies that $T_{t}$ is either a path in the case of $q_{t}=1$, or a path sticking $q_{t}$ pendent vertices at its one end, i.e., $T_{t}=S_{m_{t}}\left(q_{t}\right)$ in the case of $q_{t}>1$.

Next we show that $T_{i} \neq v_{i}$ for any $1 \leq i \leq r$. Since otherwise, $T_{1}=v_{1}$, i.e., $u v_{1}$ is a pendent edge. Note that $n>q+1$, there is $v_{i}$ such that $d_{T}\left(v_{i}\right) \geq 2$. If such a $v_{i}$ is unique then $v_{i}=v_{r}$ according to our assumption. Thus $T_{r}=S_{m_{r}}\left(q_{r}\right)$ and $T_{i}=v_{i}$ for 
$i=1,2, \ldots, r-1$. It means that $T$ is a caterpillar tree. By Theorem $4.1, T=C P_{n ;\left\lfloor\frac{q}{2}\right\rfloor,\left\lceil\frac{q}{2}\right\rceil}$ as our required. We may further assume that $d_{T}\left(v_{r-1}\right), d_{T}\left(v_{r}\right) \geq 2$, and thus $\left|T_{r-1}\right|+\left|T_{r}\right| \leq n-2$. Without loss of generality, let $\left|T_{r}\right|<\left\lfloor\frac{n}{2}\right\rfloor$. We can construct $T^{\prime \prime}=T-u v_{1}+v_{r} v_{1} \in \mathcal{T}_{n}(q)$ and define $\varphi: E(T) \longrightarrow E\left(T^{\prime \prime}\right)$ such that

$$
\varphi(e)= \begin{cases}e, & \text { if } e \neq u v_{1} \text { is any edge of } T \\ v_{r} v_{1}, & \text { if } e=u v_{1}\end{cases}
$$

It is clear that $\mu_{T}(e)=\mu_{T^{\prime \prime}}(\varphi(e))$ if $e \neq u v_{r}$. Therefore, $T$ and $T^{\prime \prime}$ are $(\varphi, \mu)$-similar with respect to $e_{1}=u v_{r}$, and $\varphi\left(e_{1}\right)=e_{1}$. Additionally, since $u$ is a centroidal vertex of $T$, we have

$$
\mu_{T^{\prime \prime}}\left(e_{1}\right)=\min \left\{\left|T_{r}\right|+1, n-\left(\left|T_{r}\right|+1\right)\right\}=\left|T_{r}\right|+1=\mu_{T}\left(u v_{r}\right)+1>\mu_{T}\left(u v_{r}\right)=\mu_{T}\left(e_{1}\right) .
$$

Therefore, $T<T^{\prime \prime}$ by Lemma3.1(1). This contradicts the maximum hypothesis of $T$.

At last we show that $r=2$. Then $T=C P_{n ;\left\lfloor\frac{q}{2}\right\rfloor,\left\lceil\frac{q}{2}\right\rceil}$ by Lemma 4.1, it is all right. Otherwise, let $r \geq 3$. Let $T^{\prime \prime \prime}$ be the tree obtained from $T$ by deleting the edge $u v_{r}$ and adding the edge $v_{1} v_{r}$. Clearly, $T^{\prime \prime \prime} \in \mathcal{T}_{n}(q)$. Now, we define bijection $\varphi: E(T) \longrightarrow E\left(T^{\prime \prime \prime}\right)$ such that

$$
\varphi(e)= \begin{cases}e, & \text { if } e \neq u v_{r} \text { is any edge of } T \\ v_{1} v_{r}, & \text { if } e=u v_{r} .\end{cases}
$$

It is clear that $\mu_{T}(e)=\mu_{T^{\prime \prime \prime}}(\varphi(e))$ if $e \neq u v_{1}$. Therefore, $T$ and $T^{\prime \prime \prime}$ are $(\varphi, \mu)$-similar with respect to $e_{1}=u v_{1}$, and $\varphi\left(e_{1}\right)=e_{1}$. Recall that $\left|T_{1}\right|=\min \left\{\left|T_{i}\right|, i=1,2, \ldots, r\right\}$, we have

$$
\mu_{T^{\prime \prime \prime}}\left(e_{1}\right)=\min \left\{\left|T_{r}\right|+\left|T_{1}\right|, \sum_{i=2}^{r-1}\left|T_{i}\right|+1\right\}>\left|T_{1}\right|=\mu_{T}\left(e_{1}\right) .
$$

Therefore, $T \prec T^{\prime \prime \prime}$ by Lemma 3.1(1), a contradiction again.

We complete this proof.

Theorem 6.1 concludes that $C P_{n ;\left\lfloor\frac{q}{2}\right\rfloor,\left\lceil\frac{q}{2}\right\rceil}$ is the maximum graph in $\left\langle\mathcal{T}_{n}(q), \leq\right\rangle$ for $2<q<$ $n-1$. By Lemma 5.1, these maximum graphs can be ordered in the following corollary.

Corollary 6.1. $S_{n}=C P_{n ;\left\lfloor\frac{n-1}{2}\right\rfloor,\left\lceil\frac{n-1}{2}\right\rceil} \prec C P_{n ;\left\lfloor\frac{n-2}{2}\right\rfloor,\left\lceil\frac{n-2}{2}\right\rceil} \prec \cdots<C P_{n ; 2,2} \prec C P_{n ; 1,2} \prec C P_{n ; 1,1}=$ $P_{n}$.

\subsection{Minimum graph in the order $\operatorname{set}\left\langle\mathcal{T}_{n}(q), \leq\right\rangle$}

Let $T$ be the starlike tree with center vertex $u$ and $v_{1}, v_{2}, \ldots, v_{q}$ be the neighbors of $u$. Then $P_{i}=P_{v_{i}}\left(v_{i} u\right)$ is a path component of $T-v_{i} u$ containing $v_{i}$. Such a $T$ we call the balanced starlike tree, denote by $S P_{n, q}$, if $\left|P_{j}\right|-\left|P_{i}\right| \leq 1$ for any pair of $1 \leq i, j \leq q$. We next prove that $S P_{n, q}$ is the unique minimum graph in $\left\langle\mathcal{T}_{n}(q), \leq\right\rangle$.

Theorem 6.2. Let $T \in \mathcal{T}_{n}(q)$, where $2<q<n-1$. Then $T \geq S P_{n, q}$, with equality if and only if $T=S P_{n, q}$. 
Proof. Let $T$ be a minimum graph in $\left\langle\mathcal{T}_{n}(q), \preceq\right\rangle$. We will show that $T=S P_{n, q}$ in what follows. We may assume that $u$ is a centroidal vertex of $T$ by Lemma 2.1(1). We will prove the following Claim 1 and Claim 2, which, put together, will get our proof.

Claim 1. $T$ is a starlike tree with centre vertex $u$.

It only needs to show that $T$ has the unique branching vertex $u$. By the way of contradiction, let $x_{1} \neq u \in V(T)$ with $d_{T}\left(x_{1}\right) \geq 3$. Without loss of generality, we assume that $P=u \cdots x_{0} x_{1} x_{2}$ is a path in $T$, where $x_{0}$ may be equal to $u$. We may chose $y_{1} \in N\left(x_{1}\right) \backslash P$ due to $d_{T}\left(x_{1}\right) \geq 3$. Let $T_{y_{1}}\left(x_{1} y_{1}\right)$ be the component of $T-x_{1} y_{1}$ containing $y_{1}$. We can construct $T^{\prime}$ obtained from $T$ by deleting edge $x_{1} y_{1}$ and adding $x_{0} y_{1}$. Clearly, $T^{\prime} \in \mathcal{T}_{n}(q)$, and we now define $\varphi: E(T) \longrightarrow E\left(T^{\prime}\right)$ such that

$$
\varphi(e)= \begin{cases}e, & \text { if } e \neq x_{1} y_{1} \text { is any edge of } T \\ x_{0} y_{1}, & \text { if } e=x_{1} y_{1}\end{cases}
$$

It is clear that $\mu_{T}(e)=\mu_{T^{\prime}}(\varphi(e))$ if $e \neq x_{0} x_{1}$. Therefore, $T$ and $T^{\prime}$ are $(\varphi, \mu)$-similar with respect to $e_{1}=x_{0} x_{1}$, and $\varphi\left(e_{1}\right)=e_{1}$. Since $u$ is a centroidal vertex, we have

$$
\mu_{T^{\prime}}\left(e_{1}\right)=\mu_{T}\left(x_{0} x_{1}\right)-\left|T_{y_{1}}\left(x_{1} y_{1}\right)\right|<\mu_{T}\left(x_{0} x_{1}\right)=\mu_{T}\left(e_{1}\right) .
$$

Therefore, $T>T^{\prime}$ by Lemma 3.1(2). This contradicts the minimum hypothesis of $T$.

Claim 2. $T=S P_{n, q}$.

According to Claim 1, we may assume that $T$ is the starlike tree with center vertex $u$ and $v_{1}, v_{2}, \ldots, v_{q}$ be the neighbors of $u$. Let $P_{i}=P_{v_{i}}\left(v_{i} u\right)$ be the component of $T-v_{i} u$ containing $v_{i}$. Then each $P_{i}$ is a path.

If we show that $\left|P_{j}\right|-\left|P_{i}\right| \leq 1$ for $1 \leq i, j \leq q$, then $T=S P_{n, q}$, it is all right. Otherwise, without loss of generality, we assume that $\left|P_{i_{0}-1}\right|+2 \leq\left|P_{i_{0}}\right|$ for some $1<i_{0} \leq q$. Now let $T^{\prime}$ be the tree obtained from $T$ by deleting the edges $u v_{i}$ and adding the edges $v_{i_{0}} v_{i}$ for $i \neq i_{0}-1, i_{0}$. Clearly, $T^{\prime} \in \mathcal{T}_{n}(q)$. We define $\varphi: E(T) \longrightarrow E\left(T^{\prime}\right)$ such that

$$
\varphi(e)= \begin{cases}e, & \text { if } e \neq u v_{i} \text { is any edge of } T \text { for } i=1, \ldots, i_{0}-2, i_{0}+1, \ldots, q \\ v_{i_{0}} v_{i}, & \text { if } e=u v_{i} \text { for } i=1, \ldots, i_{0}-2, i_{0}+1, \ldots, q\end{cases}
$$

It is clear that $\mu_{T}(e)=\mu_{T^{\prime}}(\varphi(e))$ if $e \neq u v_{i_{0}}$. Therefore, $T$ and $T^{\prime}$ are $(\varphi, \mu)$-similar with respect to $e_{1}=u v_{i_{0}}$, and $\varphi\left(e_{1}\right)=e_{1}$. Additionally, we have

$$
\mu_{T^{\prime}}\left(e_{1}\right)=\min \left\{\sum_{i \neq i_{0}-1}\left|P_{i}\right|,\left|P_{i_{0}-1}\right|+1\right\}<\left|P_{i_{0}}\right|=\mu_{T}\left(e_{1}\right) .
$$

Therefore, $T>T^{\prime}$ by Lemma 3.1(2), a contradiction.

We complete this proof.

Let $\mathcal{T}_{n}^{\Delta}$ be a set of trees with order $n$ and maximum degree $\Delta \geq 3$. In the next section, we will consider the maximal graph in $\left\langle\mathcal{T}_{n}^{\Delta}, \leq\right\rangle$. 


\section{Maximum graph in the order set $\left\langle\mathcal{T}_{n}^{\Delta}, \leq\right\rangle$}

In this section, we will give the maximum graph in the order set $\left\langle\mathcal{T}_{n}^{\Delta}, \leq\right\rangle$. If $\Delta=n-1$ then $\mathcal{T}_{n}^{\Delta}$ contains exactly one $S_{n}$, which is both maximum and minimum graph in the order set $\left\langle\mathcal{T}_{n}^{n-1}, \leq\right\rangle$. Similarly if $\Delta=2$ then $T$ is a path. Thus we always assume in this section that $2<\Delta<n-1$.

Let $C P_{n ; 1, \Delta-1}$ be the graph obtained from a path $P_{n-\Delta}=v_{1} \cdots v_{n-\Delta}$ by adding $\Delta-1$ pendent vertices at $v_{n-\Delta}$ and one pendent vertex at $v_{1}$. We will prove that $C P_{n ; 1, \Delta-1}$ is the unique maximum graph in $\left\langle\mathcal{T}_{n}^{\Delta}, \leq\right\rangle$.

Theorem 7.1. Let $T \in \mathcal{T}_{n}^{\Delta}$ where $2<\Delta<n-1$. Then $T \leq C P_{n ; 1, \Delta-1}$, with equality if and only if $T=C P_{n ; 1, \Delta-1}$.

Proof. Let $T$ be a maximum graph in $\left\langle\mathcal{T}_{n}^{\Delta}, \leq\right\rangle$. By Lemma2.1(1), we may assume that $u$ is a centroidal vertex of $T$ and show that $T=C P_{n ; 1, \Delta-1}$. In the following, we need only to show the Claim 1 and Claim 2, which, put together, will get our result.

Claim 1. $T$ is a starlike tree.

First assume that centroidal vertex $u$ is of maximum vertex and will show that $T$ is a starlike tree with centre $u$. By the way of contradiction, let $x_{1} \neq u \in V(T)$ with $d_{T}\left(x_{1}\right) \geq 3$, and $P=u \cdots x_{1}$ is a path in $T$. Denote by $D_{1}(P)$ the set of vertices such that $x_{2} \in D_{1}(P)$ iff $P \cdot x_{2}=u \cdots x_{1} x_{2}$ is a path of $T$, by $D_{2}(P)$ we mean that $x_{3} \in D_{2}(P)$ iff $P \cdot x_{2} x_{3}=u \cdots x_{1} x_{2} x_{3}$ is a path of $T$ for any $x_{3} \in D_{2}(P)$ where $x_{2} \in D_{1}(P)$, and $D_{k}(P)$ is similarly defined for $k \geq 3$.

By definition, $D_{1}(P) \neq \emptyset$. We choose any $x_{2} \in D_{1}(P)$ and let $P_{1}=P \cdot x_{2}=u \cdots x_{1} x_{2}$ be a path of $T$. Also we may select $y_{1} \in N\left(x_{1}\right) \backslash P_{1}$ due to $d_{T}\left(x_{1}\right) \geq 3$. Let $T_{y_{1}}\left(x_{1} y_{1}\right)$ be the component of $T-x_{1} y_{1}$ containing $y_{1}$. We can construct $T^{\prime}$ obtained from $T$ by deleting edge $x_{1} y_{1}$ and adding $x_{2} y_{1}$. Now we first show that $d_{T}\left(x_{2}\right)=\Delta$. Since otherwise, $T^{\prime} \in \mathcal{T}_{n}^{\Delta}$, and we will define $\varphi: E(T) \longrightarrow E\left(T^{\prime}\right)$ such that

$$
\varphi(e)= \begin{cases}e, & \text { if } e \neq x_{1} y_{1} \text { is any edge of } T \\ x_{2} y_{1}, & \text { if } e=x_{1} y_{1}\end{cases}
$$

It is clear that $\mu_{T}(e)=\mu_{T^{\prime}}(\varphi(e))$ if $e \neq x_{1} x_{2}$. Therefore, $T$ and $T^{\prime}$ are $(\varphi, \mu)$-similar with respect to $e_{1}=x_{1} x_{2}$, and $\varphi\left(e_{1}\right)=e_{1}$. Additionally, since $u$ is a centroidal vertex of $T$ on $P$ and $x_{1} \neq u$, we have

$$
\mu_{T^{\prime}}\left(e_{1}\right)=\mu_{T}\left(x_{1} x_{2}\right)+\left|T_{y_{1}}\left(x_{1} y_{1}\right)\right|>\mu_{T}\left(x_{1} x_{2}\right)=\mu_{T}\left(e_{1}\right) .
$$

Therefore, $T \prec T^{\prime}$ by Lemma 3.1 (1). This contradicts the maximum hypothesis of $T$. This also implies that every vertex of $D_{1}(P)$ has maximum degree $\Delta$ since $x_{2}$ is an arbitrary vertex in $D_{1}(P)$. Next we show that $d_{T}\left(x_{3}\right)=\Delta$ for $x_{3} \in D_{2}(P)$. In fact, $P_{1}$ can be extended as $P_{2}=u \cdots x_{1} x_{2} x_{3}$ where $x_{2} \in D_{1}(P)$. Let $y_{2} \in N\left(x_{2}\right) \backslash P_{2}$. We can construct $T^{\prime \prime}$ obtained from $T$ by deleting $x_{2} y_{2}$ and adding $x_{3} y_{2}$. Clearly, if $d_{T}\left(x_{3}\right)<\Delta$ then $T^{\prime \prime} \in \mathcal{T}_{n}^{\Delta}$, and then we define $\varphi: E(T) \longrightarrow E\left(T^{\prime \prime}\right)$ such that

$$
\varphi(e)= \begin{cases}e, & \text { if } e \neq x_{2} y_{2} \text { is any edge of } T \\ x_{3} y_{2}, & \text { if } e=x_{2} y_{2} .\end{cases}
$$


By regarding $x_{2}$ as $x_{1}$ and $y_{2}$ as $y_{1}$, we would get $T<T^{\prime \prime}$ as above arguments, a contradiction again. Similarly we can show that $d_{T}\left(x_{4}\right)=\Delta$ for $x_{4} \in D_{3}(P)$, and so on. This is impossible since this procedure can not be terminated and so $T$ is a starlike tree with centre $u$. The above proof also implies that $T$ cannot contain two vertices of degree $\Delta$.

Next assume that $d(u)<\Delta$, we will show that $d(u)=2$. Since $T \in \mathcal{T}_{n}^{\Delta}, T$ has a unique vertex of maximum degree, say $v \neq u$ with $d(v)=\Delta$ according to the above arguments. If there is some $x_{1} \neq v, u$ such that $3 \leq d\left(x_{1}\right)<\Delta$, then, as the same arguments, we would get that $d\left(x_{k}\right)=\Delta$ for $k \geq 2$, where $P_{k-1}=P \cdot x_{2} \cdots x_{k}$ and $x_{k} \in D_{k-1}(P)$. Thus $T$ is starlike tree with centre $v$ if $d(u)=2$. Otherwise $3 \leq d(u)<\Delta$ and $d(x) \leq 2$ for any $x \neq v, u$. Thus there exists a path $H=u_{1} u_{2} \cdots u_{m}$ such that $u_{t}=u$ where $2 \leq t \leq m-1$. Without loss of generality, assume that $t \leq\left\lceil\frac{m}{2}\right\rceil$. Denote by $e_{i}=u_{i} u_{i+1}$ the edges on $H$ for $i=1,2, \ldots, m-1$, we construct a tree $T^{*}$ that is obtained from $T$ by deleting edge $e_{1}=u_{1} u_{2}$ and adding a new edge $e_{m}=u_{1} u_{m}$. Now we define $\varphi: E(T) \longrightarrow E\left(T^{*}\right)$ such that

$$
\varphi(e)= \begin{cases}e, & \text { if } e \neq u_{i} u_{i+1} \text { for } i=1,2, \ldots, m-1 \\ u_{i+1} u_{i+2}, & \text { for } e_{i}=u_{i} u_{i+1} \text { and } i=1,2, \ldots, m-2 \\ u_{1} u_{m}, & \text { for } e_{m-1}=u_{m-1} u_{m}\end{cases}
$$

It is easy to verify that $\mu_{T}(e)=\mu_{T^{\prime}}(\varphi(e))$ if $e \neq u_{t-1} u_{t}$. Therefore, $T$ and $T^{*}$ are $(\varphi, \mu)$ similar with respect to $e_{t-1}=u_{t-1} u_{t}$, and $\varphi\left(e_{t-1}\right)=e_{t}$. Recall that $u_{t}=u$ is centroidal vertex, we have $\mu_{T}\left(e_{t-1}\right)=\mu_{T}\left(u_{t-1} u_{t}\right)=t-1<m-t+1=\mu_{T^{*}}\left(u_{t} u_{t+1}\right)=\mu_{T^{*}}\left(e_{t}\right)$, then $T \prec T^{*}$ by Lemma 3.1(1), a contradiction. Therefore, $d(u)=2$ and $T$ is a starlike tree with centre $v$.

Claim 2. $T=C P_{n ; 1, \Delta-1}$.

According to Claim 1, we may assume that $T$ is the starlike tree with centre vertex $v$ and $v_{1}, v_{2}, \ldots, v_{\Delta}$ be the neighbors of $v$. Let $P_{i}=P_{v_{i}}\left(v_{i} v\right)$ be the component of $T-v_{i} v$ containing $v_{i}$. Without loss of generality, we assume that $\left|P_{i}\right| \leq\left|P_{j}\right|$ for $i \leq j$.

If we show that $\left|P_{1}\right|=\cdots=\left|P_{\Delta-1}\right|=1$, then $T=C P_{n ; 1, \Delta-1}$, it is all right. Otherwise, let $\left|P_{\Delta}\right| \geq\left|P_{\Delta-1}\right| \geq 2$. Let $T^{\prime \prime \prime}$ be the tree obtained from $T$ by deleting the edges $v v_{i}$ and adding the edges $v_{\Delta-1} v_{i}$ for $i=1,2, \ldots, \Delta-2$. Clearly, $T^{\prime \prime \prime} \in \mathcal{T}_{n}^{\Delta}$. We define $\varphi: E(T) \longrightarrow E\left(T^{\prime \prime \prime}\right)$ such that

$$
\varphi(e)= \begin{cases}e, & \text { if } e \neq v v_{i} \text { is any edge of } T \text { for } i=1, \ldots, \Delta-2, \\ v_{\Delta-1} v_{i}, & \text { if } e=v v_{i} \text { for } i=1, \ldots, \Delta-2\end{cases}
$$

It is clear that $\mu_{T}(e)=\mu_{T^{\prime \prime \prime}}(\varphi(e))$ if $e \neq v v_{\Delta-1}$. Therefore, $T$ and $T^{\prime \prime \prime}$ are $(\varphi, \mu)$-similar with respect to $e_{1}=v v_{\Delta-1}$, and $\varphi\left(e_{1}\right)=e_{1}$. Additionally, note that $u$ is centroidal vertex including in $\{v\} \cup V\left(P_{\Delta}\right)$ due to $\left|P_{\Delta}\right| \geq\left|P_{\Delta-1}\right| \geq 2$, we have

$$
\mu_{T^{\prime \prime \prime}}\left(e_{1}\right)=\min \left\{\sum_{i=1}^{\Delta-1}\left|P_{i}\right|,\left|P_{\Delta}\right|+1\right\}>\left|P_{\Delta-1}\right|=\mu_{T}\left(e_{1}\right) .
$$

Therefore, $T \prec T^{\prime \prime \prime}$ by Lemma3.1(1), a contradiction.

We complete this proof.

According to Theorem 7.1, given $n$ we know that $T=C P_{n ; 1, \Delta-1}$ is maximum graph in 
$\left\langle\mathcal{T}_{n}^{\Delta}, \leq\right\rangle$ for $2<\Delta<n-1$. By Lemma 5.1, these maximum graphs can be ordered in the following corollary.

Corollary 7.1. $S_{n}=C P_{n ; 1, n-2}<C P_{n ; 1, n-3} \prec \cdots \prec C P_{n ; 1,3}<C P_{n ; 1,2}<C P_{n ; 1,1}=P_{n}$.

Remark 7.1. From Theorem 6.2, we know that $S P_{n, q}$ is minimum graph in $\left\langle\mathcal{T}_{n}(q), \leq\right\rangle$. However, the minimum graph in $\left\langle\mathcal{T}_{n}^{\Delta}, \leq\right\rangle$ leaves unknown.

\section{Application}

It is well known that topological index of a graph is usually defined as a function of distance, such as Wiener index of a graph $G: W(G)=\sum_{\{u, v\} \in V(G)} d(u, v)$. For a pair of vertices $\{u, v\}$ in a tree $T \in \mathcal{T}_{n}$, there is a unique path $P_{u, v}$ connecting them with exactly $d(u, v)$ edges. Thus $W(T)$ can be represented by a function of $\mu(e)$ as follows:

$$
\begin{aligned}
W(T) & =\sum_{\{u, v\} \in V(T)} d(u, v)=\sum_{\{u, v\} \in V(T)}\left|E\left(P_{u, v}\right)\right| \\
& =\sum_{e=x y \in E(T)} n_{x}(e) n_{y}(e)=\sum_{e=x y \in E(T)} n_{x}(e)\left(n-n_{x}(e)\right) \\
& =\sum_{e \in E(T)} \mu(e)(n-\mu(e)) .
\end{aligned}
$$

Let $\mathbf{r}=\left(r_{1}, r_{2}, \ldots, r_{\left\lfloor\frac{n}{2}\right\rfloor}\right)$ be the edge division vector of $T$ and $f(x)=x(n-x)$. As in the proof of Theorem 8 in [1], $W(T)$ can be further simplified as

$$
W(T)=\sum_{e \in E(T)} \mu(e)(n-\mu(e))=\sum_{1 \leq i \leq\left\lfloor\frac{n}{2}\right\rfloor} r_{i} f(i) .
$$

Since there are large part of topological indices of a graph can be also represented by some functions of $\mu(e)$ described as Eq. (3), the authors in [1] introduced the notions bellow.

Definition 8.1. Let $F: \mathcal{T}_{n} \rightarrow \mathbb{R}$ be a topological index and let $f: \mathbb{N} \rightarrow \mathbb{R}$ be a real function defined for positive integers. The topological index $F$ is an edge additive eccentric topological index if it holds that $F(G)=\sum_{e \in E(G)} f(\mu(e))$. Function $f$ is called the edge contribution function of index $F$.

Let $F$ be the edge additive eccentric topological index and $T, T^{\prime} \in \mathcal{T}_{n}$. From Eq. (3), the authors in [1] gave that

$$
\begin{aligned}
F\left(T^{\prime}\right)-F(T)= & \sum_{1 \leq i \leq\left\lfloor\frac{n}{2}\right\rfloor}\left(r_{i}^{\prime}-r_{i}\right) f(1)+\sum_{2 \leq i \leq\left\lfloor\frac{n}{2}\right\rfloor}\left(r_{i}^{\prime}-r_{i}\right)(f(2)-f(1))+\cdots \\
& +\sum_{\left\lfloor\frac{n}{2}\right\rfloor \leq i \leq\left\lfloor\frac{n}{2}\right\rfloor}\left(r_{i}^{\prime}-r_{i}\right)\left(f\left(\left\lfloor\frac{n}{2}\right\rfloor\right)-f\left(\left\lfloor\frac{n}{2}\right\rfloor-1\right)\right),
\end{aligned}
$$

Eq. (4) implies that $F(T) \leq F\left(T^{\prime}\right)$ (resp., $F(T)<F\left(T^{\prime}\right)$ ) if $T<T^{\prime}$ and $f(x)$ is increasing (resp., proper increasing). Such an index $F$ is defined in [1] to be of Wiener type if $f(x)$ is increasing, and of anti-Wiener type if $f(x)$ is decreasing. The above idea provides of a new method to find the extremal graphs and evolute the bounds with respect to topological index $F$. Summarizing the above arguments leads to the following result.

Theorem 8.1. Let $\left\langle\mathcal{H}_{n}, \leq\right\rangle$ be an order subset of $\left\langle\mathcal{T}_{n}, \leq\right\rangle$ and $H$ be an minimum (maximum) graph of $\left\langle\mathcal{H}_{n}, \leq\right\rangle$. Let $F: \mathcal{H}_{n} \longrightarrow \mathbb{R}$ be an edge additive eccentric topological index. We 
have

(1) If $F$ is an index of Wiener type, then $H$ is also an minimum (maximum) graph of $\mathcal{H}_{n}$ with respect to $F$.

(2) If $F$ is an index of anti-Wiener type, then $H$ is maximum (minimum) graph of $\mathcal{H}_{n}$ with respect to $F$.

By applying Theorem 8.1, the authors in [1] (Theorem 12,17) proved the conclusions that we summarize in the Table 1.

Table 1: Some conclusions of indices

\begin{tabular}{|c|c|c|c|c|}
\hline Indices & Definition & Edge contribution function & Type & Extremal graphs in $\mathcal{T}_{n}$ \\
\hline $\begin{array}{l}\text { Wiener } \\
\text { index }\end{array}$ & $\begin{array}{c}W(G)= \\
\sum_{\{u, v\} \in V(G)} d(u, v)\end{array}$ & $f(x)=x(n-x)$ & Wiener type & $W\left(S_{n}\right) \leq W(T) \leq W\left(P_{n}\right)$ \\
\hline $\begin{array}{l}\text { Modified } \\
\text { Wiener } \\
\text { indices }\end{array}$ & $\begin{array}{c}{ }^{\lambda} W(G)= \\
\sum_{\{u, v\} \in V(G)} d^{\lambda}(u, v)\end{array}$ & $f(x)=x^{\lambda}(n-x)^{\lambda}$ & $\begin{array}{c}\text { Wiener type for } \\
\lambda>0 \text { and anti-Wiener } \\
\text { type for } \lambda<0\end{array}$ & $\begin{array}{c}{ }^{\lambda} W\left(S_{n}\right) \leq{ }^{\lambda} W(T) \leq^{\lambda} W\left(P_{n}\right) \\
\text { for } \lambda>0 \text { and } \\
{ }^{\lambda} W\left(P_{n}\right) \leq{ }^{\lambda} W(T) \leq^{\lambda} W\left(S_{n}\right) \\
\text { for } \lambda<0\end{array}$ \\
\hline $\begin{array}{l}\text { Variable } \\
\text { Wiener } \\
\text { indices }\end{array}$ & $\begin{array}{c}\lambda W(G)= \\
\frac{1}{2} \sum_{e=u v \in E(G)}\left(n^{\lambda}-\right. \\
\left.n_{u}(e)^{\lambda}-n_{v}(e)^{\lambda}\right)\end{array}$ & $f(x)=n^{\lambda}-x^{\lambda}-(n-x)^{\lambda}$ & $\begin{array}{l}\text { Wiener type for } \\
\lambda>1 \text { and anti-Wiener } \\
\text { type for } \lambda<1\end{array}$ & $\begin{array}{c}{ }_{\lambda} W\left(S_{n}\right) \leq_{\lambda} W(T) \leq_{\lambda} W\left(P_{n}\right) \\
\text { for } \lambda>1 \text { and } \\
{ }_{\lambda} W\left(P_{n}\right) \leq_{\lambda} W(T) \leq_{\lambda} W\left(S_{n}\right) \\
\quad \text { for } \lambda<1\end{array}$ \\
\hline $\begin{array}{l}\text { Steiner } \\
k \text {-Wiener } \\
\text { index }\end{array}$ & $\begin{array}{c}S W_{k}(G)= \\
\sum_{e=u v \in E(G)} \sum_{i=1}^{k-1} \\
\left(\begin{array}{c}n_{u}(e) \\
i\end{array}\right)\left(\begin{array}{c}n_{v}(e) \\
k-i\end{array}\right)\end{array}$ & $f(x)=\left(\begin{array}{l}n \\
k\end{array}\right)-\left(\begin{array}{l}x \\
k\end{array}\right)-\left(\begin{array}{c}n-x \\
k\end{array}\right)$ & Wiener type & $S W_{k}\left(S_{n}\right) \leq S W_{k}(T) \leq S W_{k}\left(P_{n}\right)$ \\
\hline
\end{tabular}

As similar as the above conclusions, we will give the extremal graphs with respect to the topological indices of hyper-Wiener index, Wiener-Hosoya index, degree distance, Gutman index and $A B C_{2}$ index, respectively. It suffices to verify that wether these indices are edge additive eccentric topological index $F(G)$ and wether the corresponding edge contribution function $f$ is monotonous such that $F(G)=\sum_{e \in E(G)} f(\mu(e))$.

It is easy to verify that hyper-Wiener index can be written as

$$
\begin{aligned}
W W(T) & =\sum_{e=u v \in E(T)}\left(\frac{1}{2} n_{u}(e) n_{v}(e)+\frac{1}{2} n_{u}(e)^{2} n_{v}(e)^{2}\right) \\
& =\sum_{e=u v \in E(T)}\left[\frac{1}{2} n_{u}(e)\left(n-n_{u}(e)\right)+\frac{1}{2} n_{u}(e)^{2}\left(n-n_{u}(e)\right)^{2}\right] \\
& =\sum_{1 \leq i \leq\left\lfloor\frac{n}{2}\right\rfloor} r_{i} f_{w w}(i)
\end{aligned}
$$

where its edge contribution function is $f_{w w}(x)=\frac{1}{2} x(n-x)+\frac{1}{2} x^{2}(n-x)^{2}$. It is routine to verify that $f_{w w}(x)$ is strictly increasing. Hence the hyper-Wiener index for tree is of Wiener type.

For $e=u v \in E(T)$, note that $h(e)=n_{u}(e) n_{v}(e)$ and $h[e]=\left(n_{u}(e)-1\right)\left(n_{v}(e)-1\right)$, the Wiener-Hosoya index can be written as

$$
\begin{aligned}
h(T) & =\sum_{e=u v \in E(T)}\left[n_{u}(e) n_{v}(e)+\left(n_{u}(e)-1\right)\left(n_{v}(e)-1\right)\right] \\
& =\sum_{e=u v \in E(T)}\left[n_{u}(e)\left(n-n_{u}(e)\right)+\left(n_{u}(e)-1\right)\left(n-n_{u}(e)-1\right)\right] \\
& =\sum_{1 \leq i \leq\left\lfloor\frac{n}{2}\right\rfloor} r_{i} f_{h}(i)
\end{aligned}
$$

where its edge contribution function is $f_{h}(x)=x(n-x)+(x-1)(n-x-1)$. It is routine to verify that $f_{h}(x)$ is strictly increasing. Hence the Wiener-Hosoya index for tree is of Wiener type. 
The degree distance can be written as

$$
\begin{aligned}
D^{\prime}(T) & =\sum_{e=u v \in E(T)}\left(4 n_{u}(e) n_{v}(e)-n\right) \\
& =\sum_{e=u v \in E(T)}\left[4 n_{u}(e)\left(n-n_{u}(e)\right)-n\right] \\
& =\sum_{1 \leq i \leq\left\lfloor\frac{n}{2}\right\rfloor} r_{i} f_{D^{\prime}}(i)
\end{aligned}
$$

where its edge contribution function is $f_{D^{\prime}}(x)=4 x(n-x)-n$. It is routine to verify that $f_{D^{\prime}}(x)$ is strictly increasing. Hence the degree distance for tree is of Wiener type.

The Gutman index can be written as

$$
\begin{aligned}
\operatorname{Gut}(T) & =\sum_{e=u v \in E(T)}\left[4 n_{u}(e) n_{v}(e)-(2 n-1)\right] \\
& =\sum_{e=u v \in E(T)}\left[4 n_{u}(e)\left(n-n_{u}(e)\right)-(2 n-1)\right] \\
& =\sum_{1 \leq i \leq\left\lfloor\frac{n}{2}\right\rfloor} r_{i} f_{G u t}(i)
\end{aligned}
$$

where its edge contribution function is $f_{G u t}(x)=4 x(n-x)-(2 n-1)$. It is routine to verify that $f_{\text {Gut }}(x)$ is strictly increasing. Hence the Gutman index for tree is of Wiener type.

The second atom-bond connectivity index can be written as

$$
\begin{aligned}
A B C_{2}(T) & =\sum_{e=u v \in E(T)} \sqrt{\frac{n-2}{n_{u}(e)\left(n-n_{u}(e)\right)}} \\
& =\sum_{e=u v \in E(T)} \sqrt{n-2} n_{u}(e)^{-\frac{1}{2}}\left(n-n_{u}(e)\right)^{-\frac{1}{2}} \\
& =\sum_{1 \leq i \leq\left\lfloor\frac{n}{2}\right\rfloor} r_{i} f_{A B C_{2}}(i)
\end{aligned}
$$

where its edge contribution function is $f_{A B C_{2}}(x)=\sqrt{n-2} x^{-\frac{1}{2}}(n-x)^{-\frac{1}{2}}$. It is routine to verify that $f_{A B C_{2}}(x)$ is strictly decreasing. Hence the second atom-bond connectivity index for tree is of anti-Wiener type.

\begin{tabular}{|c|c|c|c|c|}
\hline Type & \multicolumn{2}{|c|}{ Wiener type indices } & \multicolumn{2}{|c|}{ anti-Wiener type indices } \\
\hline Indices & \multicolumn{2}{|c|}{$W W(\cdot), h(\cdot), D^{\prime}(\cdot), \operatorname{Gut}(\cdot)$} & \multicolumn{2}{|c|}{$A B C_{2}(\cdot)$} \\
\hline Classes & $\begin{array}{l}\text { The graphs with } \\
\text { maximum indices }\end{array}$ & $\begin{array}{l}\text { The graphs with } \\
\text { minimum indices }\end{array}$ & $\begin{array}{l}\text { The graphs with } \\
\text { maximum indices }\end{array}$ & $\begin{array}{l}\text { The graphs with } \\
\text { minimum indices }\end{array}$ \\
\hline $\mathcal{T}_{n}$ & $P_{n}$ & $S_{n}$ & $S_{n}$ & $P_{n}$ \\
\hline$C(n, k)$ & $C P_{\left\lfloor\frac{n-k}{2}\right\rfloor,\left\lceil\frac{n-k}{2}\right\rceil}$ & $C P_{n, k}^{\left\lceil\frac{k}{2}\right\rceil}$ & $C P_{n, k}^{\left\lceil\frac{k}{2}\right\rceil}$ & $C P_{\left\lfloor\frac{n-k}{2}\right\rfloor,\left\lceil\frac{n-k}{2}\right\rceil}$ \\
\hline $\mathcal{T}(n, k-1)$ & & $C P_{n, k}^{\left\lceil\frac{k}{2}\right\rceil}$ & $C P_{n, k}^{\left\lceil\frac{k}{2}\right\rceil}$ & \\
\hline $\mathcal{T}_{n}(q)$ & $C P_{\left\lfloor\frac{q}{2}\right\rfloor,\left\lceil\frac{q}{2}\right\rceil}$ & $S P_{n, q}$ & $S P_{n, q}$ & $C P_{\left\lfloor\frac{q}{2}\right\rfloor\left\lceil\left\lceil\frac{q}{2}\right\rceil\right.}$ \\
\hline $\mathcal{T}_{n}^{\Delta}$ & $C P_{n ; 1, \Delta-1}$ & & & $C P_{n ; 1, \Delta-1}$ \\
\hline
\end{tabular}

By setting $\left\langle\mathcal{H}_{n}, \leq\right\rangle=\langle C(n, k), \leq\rangle,\langle\mathcal{T}(n, k-1), \leq\rangle,\left\langle\mathcal{T}_{n}(q), \leq\right\rangle$ or $\left\langle\mathcal{T}_{n}^{\Delta}, \leq\right\rangle$, we have determined their extremal graphs in Theorem 4.14.25.16.16.2 and 7.1, respectively. From the above discussions, we get the following theorem by applying Theorem 8.1 . Its conclusions are summarized in the Table 2.

Theorem 8.2. The extremal graphs in the classes $\mathcal{T}_{n}, C(n, k), \mathcal{T}(n, k-1), \mathcal{T}_{n}(q)$ and $\mathcal{T}_{n}^{\Delta}$ with respect to all the topological indices of $W W(\cdot), h(\cdot), D^{\prime}(\cdot)$, Gut( $\left.\cdot\right)$ and $A B C_{2}(\cdot)$ are listed in Table 2.

Table 2: Some conclusions of indices 
There are some sporadic results published in various literatures [13, 24, 26, 27, 29, 30] about the topological indices of a graph, such as the indices of Wiener index, hyperWiener index, Wiener-Hosoya index, degree distance, Gutman index and $A B C_{2}$ index and so on, in which the authors mainly considered the extremal graphs with respect to some topological indices on subset of trees, such as $\mathcal{T}_{n}, C(n, k), T(n, k-1), \mathcal{T}_{n}(q)$ and $\mathcal{T}_{n}^{\Delta}$. We now summarize our results of Theorem 8.1 and Theorem 8.2 along with the known results in the following Table 3 . In this table, each cell lists the extremal graphs of the class specified, in which the results marked in black are old, marked blue are new (in Theorem 8.1 and Theorem 8.2 and the question mark are still open.

The extremal graphs given in Theorem 8.2 and Table 3 all have specific structures whose topological indices can be simply calculated by the expression Eq. (3). It is clear that the values of these topological indices of extremal graphs can be used as the sharp bounds for the corresponding classes of trees. As an example we give the bounds of Wiener index in the classes $C(n, k), \mathcal{T}_{n}(q)$ and $\mathcal{T}_{n}^{\Delta}$ in the following propositions, in which the proof and calculation are omitted because they are conventional.

Proposition 8.2.1. Let $T \in C(n, k)$ where $k \geq 2$, we have

$$
W(T) \geq W\left(C P_{n, k}^{\left\lceil\frac{k}{2}\right\rceil}\right)= \begin{cases}-\frac{k^{3}}{12}+\frac{n k^{2}}{4}-k n+\frac{13 k}{12}+n^{2}-\frac{5 n}{4}, & \text { if } k \text { is odd } \\ -\frac{k^{3}}{12}+\frac{n k^{2}}{4}-k n+\frac{5 k}{6}+n^{2}-n, & \text { if } k \text { is even }\end{cases}
$$

and

$$
W(T) \leq W\left(C P_{\left\lfloor\frac{n-k}{2}\right\rfloor,\left\lceil\frac{n-k}{2}\right\rceil}\right)= \begin{cases}-\frac{k^{3}}{12}+\frac{k^{2}}{4}+\frac{k n^{2}}{4}-k n+\frac{5 k}{6}+\frac{3 n^{2}}{4}-n, & \text { if } n-k \text { is even } \\ -\frac{k^{3}}{12}+\frac{k^{2}}{4}+\frac{k n^{2}}{4}-k n+\frac{7 k}{12}+\frac{3 n^{2}}{4}-n+\frac{1}{4}, & \text { if } n-k \text { is odd. }\end{cases}
$$

Proposition 8.2.2. Let $T \in \mathcal{T}_{n}(q)$. Let $n-1=s q+r$ where $q \geq 3$ and $0 \leq r \leq q-1$, then

$$
W(T) \geq W\left(S P_{n, q}\right)=\frac{3 q-2}{6} q s^{3}+\left(\frac{1}{2} q^{2}+\frac{3}{2} q r-r\right) s^{2}+\left(r^{2}+\frac{3}{2} q r+\frac{1}{3} q-r\right) s+r^{2},
$$

and

$$
W(T) \leq W\left(C P_{\left\lfloor\frac{q}{2}\right\rfloor,\left\lceil\frac{q}{2}\right\rceil}\right)= \begin{cases}\frac{n^{3}}{6}-\frac{n q^{2}}{4}+\frac{n q}{2}-\frac{n}{6}+\frac{q^{3}}{12}+\frac{q^{2}}{4}-\frac{5 q}{6}, & \text { if } q \text { is even } \\ \frac{n^{3}}{6}-\frac{n q^{2}}{4}+\frac{n q}{2}-\frac{5 n}{12}+\frac{q^{3}}{12}+\frac{q^{2}}{4}-\frac{7 q}{12}+\frac{1}{4}, & \text { if } q \text { is odd } .\end{cases}
$$

Proposition 8.2.3. Let $T \in \mathcal{T}_{n}^{\Delta}$, we have

$$
\begin{aligned}
W(T) & \leq W\left(C P_{n ; 1, \Delta-1}\right) \\
& = \begin{cases}\frac{\Delta^{3}}{3}-\frac{n+1}{2} \Delta^{2}+\frac{9 n-5}{6} \Delta+\frac{n^{3}}{6}-\frac{7 n}{6}+1, & \text { if } \Delta<\left\lfloor\frac{n}{2}\right\rfloor \text { and } \Delta>\left\lceil\frac{n}{2}\right\rceil \\
\frac{n^{3}}{12}+\frac{5 n^{2}}{8}-\frac{19 n}{12}+1, & \text { if } n \geq 4 \text { is } \text { even } \text { and } \Delta=\frac{n}{2} \\
\frac{n^{3}}{12}+\frac{3 n^{2}}{4}-\frac{25 n}{12}+\frac{5}{4}, & \text { if } n \geq 5 \text { is odd } \text { and } \Delta=\left\lfloor\frac{n}{2}\right\rfloor \\
\frac{n^{3}}{12}+\frac{n^{2}}{2}-\frac{13 n}{12}+\frac{1}{2}, & \text { if } n \geq 5 \text { is odd and } \Delta=\left\lceil\frac{n}{2}\right\rceil .\end{cases}
\end{aligned}
$$

At last of this paper, in terms of the formulas in Proposition 8.2.1 we list the bounds of the Wiener index in the class $C(n, k)$ for $5 \leq n \leq 11$ and $4 \leq k \leq 10$ in Table 4 . 
Table 3: Some known and new results of indices

\begin{tabular}{|c|c|c|c|c|c|c|}
\hline Indices & Type & trees in $\mathcal{T}_{n}$ & trees in $C(n, k)$ & trees in $T(n, k-1)$ & trees in $\mathcal{T}_{n}(q)$ & trees in $\mathcal{T}_{n}^{\Delta}$ \\
\hline $\begin{array}{l}\text { Wiener } \\
\text { index }\end{array}$ & $\begin{array}{l}\text { minimum } \\
\text { maximum } \\
\text { references }\end{array}$ & $\begin{array}{l}S_{n} \\
P_{n} \\
{[20]}\end{array}$ & $\begin{array}{c}C P_{n, k}^{\left\lceil\frac{k}{2}\right\rceil} \\
C P_{\left\lfloor\frac{n-k}{2}\right\rfloor,\left\lceil\frac{n-k}{2}\right\rceil} \\
{[22]}\end{array}$ & $\begin{array}{c}C P_{n, k}^{\left\lceil\frac{k}{2}\right\rceil} \\
? \\
21,22\end{array}$ & $\begin{array}{c}S P_{n, q} \\
C P_{\left\lfloor\frac{q}{2}\right\rfloor,\left\lceil\frac{q}{2}\right\rceil} \\
{[23}\end{array}$ & $\begin{array}{c}? \\
C P_{n ; 1, \Delta-1} \\
{[22}\end{array}$ \\
\hline $\begin{array}{l}\text { Modified } \\
\text { Wiener } \\
\text { indices }\end{array}$ & $\begin{array}{l}\text { minimum } \\
\text { maximum } \\
\text { references }\end{array}$ & $\begin{array}{c}S_{n}(\lambda>0), P_{n}(\lambda<0) \\
P_{n}(\lambda>0), S_{n}(\lambda<0) \\
24\end{array}$ & $\begin{array}{l}C P_{n, k}^{\left\lceil\frac{k}{2}\right\rceil}(\lambda>0), C P_{\left\lfloor\frac{n-k}{2}\right\rfloor,\left\lceil\frac{n-k}{2}\right\rceil}(\lambda<0) \\
C P_{\left\lfloor\frac{n-k}{2}\right\rfloor,\left\lceil\frac{n-k}{2}\right\rceil}(\lambda>0), C P_{n, k}^{\left\lceil\frac{k}{2}\right\rceil}(\lambda<0)\end{array}$ & $\begin{array}{c}C P_{n, k}^{\left\lceil\frac{k}{2}\right\rceil}(\lambda>0), ?(\lambda<0) \\
C P_{n, k}^{\left\lceil\frac{k}{2}\right\rceil}(\lambda<0), ?(\lambda>0) \\
{[28}\end{array}$ & $\begin{array}{c}S P_{n, q}(\lambda>0), C P_{\left\lfloor\frac{q}{2}\right\rfloor,\left\lceil\frac{q}{2}\right\rceil}(\lambda<0) \\
C P_{\left\lfloor\frac{q}{2}\right\rfloor,\left\lceil\frac{q}{2}\right\rceil}(\lambda>0), S P_{n, q}(\lambda<0) \\
29\end{array}$ & $\begin{array}{c}C P_{n ; 1, \Delta-1}(\lambda<0), ?(\lambda>0) \\
C P_{n ; 1, \Delta-1}(\lambda>0), ?(\lambda<0) \\
26,27\end{array}$ \\
\hline $\begin{array}{l}\text { Variable } \\
\text { Wiener } \\
\text { indices }\end{array}$ & $\begin{array}{l}\text { minimum } \\
\text { maximum } \\
\text { references }\end{array}$ & $\begin{array}{c}S_{n}(\lambda>1), P_{n}(\lambda<1) \\
P_{n}(\lambda>1), S_{n}(\lambda<1) \\
30\end{array}$ & $\begin{array}{l}C P_{n, k}^{\left\lceil\frac{k}{2}\right\rceil}(\lambda>1), C P_{\left\lfloor\frac{n-k}{2}\right\rfloor\left\lceil\frac{n-k}{2}\right\rceil}(\lambda<1) \\
C P_{\left\lfloor\frac{n-k}{2}\right\rfloor,\left\lceil\frac{n-k}{2}\right\rceil}(\lambda>1), C P_{n, k}^{\left\lceil\frac{k}{2}\right\rceil}(\lambda<1)\end{array}$ & $\begin{array}{l}C P_{n, k}^{\left\lceil\frac{k}{2}\right\rceil}(\lambda>1) \\
C P_{n, k}^{\left\lceil\frac{k}{2}\right\rceil}(\lambda<1)\end{array}$ & $\begin{array}{l}S P_{n, q}(\lambda>1), C P_{\left\lfloor\frac{q}{2}\right\rfloor,\left\lceil\frac{q}{2}\right\rceil}(\lambda<1) \\
C P_{\left\lfloor\frac{q}{2}\right\rfloor,\left\lceil\frac{q}{2}\right\rceil}(\lambda>1), S P_{n, q}(\lambda<1)\end{array}$ & $\begin{array}{l}C P_{n ; 1, \Delta-1}(\lambda<1) \\
C P_{n ; 1, \Delta-1}(\lambda>1)\end{array}$ \\
\hline $\begin{array}{l}\text { Steiner } \\
k \text {-Wiener } \\
\text { index }\end{array}$ & $\begin{array}{l}\text { minimum } \\
\text { maximum } \\
\text { references }\end{array}$ & $\begin{array}{l}S_{n} \\
P_{n} \\
112\end{array}$ & $\begin{array}{c}C P_{n, k}^{\left\lceil\frac{k}{2}\right\rceil} \\
C P_{\left\lfloor\frac{n-k}{2}\right\rfloor,\left\lceil\frac{n-k}{2}\right\rceil}\end{array}$ & $\begin{array}{c}C P_{n, k}^{\left\lceil\frac{k}{2}\right\rceil} \\
? \\
{[13} \\
\end{array}$ & $\begin{array}{c}S P_{n, q} \\
C P_{\left\lfloor\frac{q}{2}\right\rfloor,\left\lceil\frac{q}{2}\right\rceil} \\
114\end{array}$ & $\begin{array}{c}? \\
C P_{n ; 1, \Delta-1} \\
{[14}\end{array}$ \\
\hline $\begin{array}{c}\text { hyper } \\
\text {-Wiener } \\
\text { index }\end{array}$ & $\begin{array}{l}\text { minimum } \\
\text { maximum } \\
\text { references }\end{array}$ & $\begin{array}{c}S_{n} \\
P_{n} \\
15 \\
\end{array}$ & $\begin{array}{c}C P_{n, k}^{\left\lceil\frac{k}{2}\right\rceil} \\
C P_{\left\lfloor\frac{n-k}{2}\right\rfloor,\left\lceil\frac{n-k}{2}\right\rceil}\end{array}$ & $\begin{array}{c}C P_{n, k}^{\left\lceil\frac{k}{2}\right\rceil} \\
? \\
16,17 \\
\end{array}$ & $\begin{array}{c}S P_{n, q} \\
C P_{\left\lfloor\frac{q}{2}\right\rfloor,\left\lceil\frac{q}{2}\right\rceil} \\
116\end{array}$ & $\begin{array}{c}? \\
C P_{n ; 1, \Delta-1} \\
16\end{array}$ \\
\hline $\begin{array}{c}\text { Wiener } \\
\text {-Hosoya } \\
\text { index }\end{array}$ & $\begin{array}{l}\text { minimum } \\
\text { maximum } \\
\text { references }\end{array}$ & $\begin{array}{l}S_{n} \\
P_{n} \\
18\end{array}$ & $\begin{array}{c}C P_{n, k}^{\left\lceil\frac{k}{2}\right\rceil} \\
C P_{\left\lfloor\frac{n-k}{2}\right\rfloor,\left\lceil\frac{n-k}{2}\right\rceil}\end{array}$ & $\begin{array}{c}C P_{n, k}^{\left\lceil\frac{k}{2}\right\rceil} \\
? \\
18\end{array}$ & $\begin{array}{c}S P_{n, q} \\
C P_{\left\lfloor\frac{q}{2}\right\rfloor,\left\lceil\frac{q}{2}\right\rceil}\end{array}$ & $\begin{array}{c}? \\
C P_{n ; 1, \Delta-1}\end{array}$ \\
\hline $\begin{array}{c}\text { degree } \\
\text { distance }\end{array}$ & $\begin{array}{l}\text { minimum } \\
\text { maximum } \\
\text { references }\end{array}$ & $\begin{array}{c}S_{n} \\
P_{n} \\
19\end{array}$ & $\begin{array}{c}C P_{n, k}^{\left\lceil\frac{k}{2}\right\rceil} \\
C P_{\left\lfloor\frac{n-k}{2}\right\rfloor,\left\lceil\frac{n-k}{2}\right\rceil}\end{array}$ & $\begin{array}{c}C P_{n, k}^{\left\lceil\frac{k}{2}\right\rceil} \\
?\end{array}$ & $\begin{array}{c}S P_{n, q} \\
C P_{\left\lfloor\frac{q}{2}\right\rfloor,\left\lceil\frac{q}{2}\right\rceil}\end{array}$ & $\begin{array}{c}? \\
C P_{n ; 1, \Delta-1}\end{array}$ \\
\hline $\begin{array}{c}\text { Gutman } \\
\text { index }\end{array}$ & $\begin{array}{l}\text { minimum } \\
\text { maximum } \\
\text { references }\end{array}$ & $\begin{array}{l}S_{n} \\
P_{n} \\
9\end{array}$ & $\begin{array}{c}C P_{n, k}^{\left\lceil\frac{k}{2}\right\rceil} \\
C P_{\left\lfloor\frac{n-k}{2}\right\rfloor,\left\lceil\frac{n-k}{2}\right\rceil}\end{array}$ & $\begin{array}{c}C P_{n, k}^{\left\lceil\frac{k}{2}\right\rceil} \\
?\end{array}$ & $\begin{array}{c}S P_{n, q} \\
C P_{\left\lfloor\frac{q}{2}\right\rfloor,\left\lceil\frac{q}{2}\right\rceil}\end{array}$ & $\begin{array}{c}? \\
C P_{n ; 1, \Delta-1}\end{array}$ \\
\hline $\begin{array}{l}A B C_{2} \\
\text { index }\end{array}$ & $\begin{array}{l}\text { minimum } \\
\text { maximum } \\
\text { references }\end{array}$ & $\begin{array}{l}P_{n} \\
S_{n} \\
8\end{array}$ & $\begin{array}{c}C P_{\left\lfloor\frac{n-k}{2}\right\rfloor,\left\lceil\frac{n-k}{2}\right\rceil} \\
C P_{n, k}^{\left\lceil\frac{k}{2}\right\rceil}\end{array}$ & $\begin{array}{c}? \\
C P_{n, k}^{\left\lceil\frac{k}{2}\right\rceil}\end{array}$ & $\begin{array}{l}C P_{\left\lfloor\frac{q}{2}\right\rfloor,\left\lceil\frac{q}{2}\right\rceil} \\
\quad S P_{n, q}\end{array}$ & $\begin{array}{c}C P_{n ; 1, \Delta-1} \\
?\end{array}$ \\
\hline
\end{tabular}


Table 4: $W\left(C P_{n, k}^{\left\lceil\frac{k}{2}\right\rceil}\right)$ and $W\left(C P_{\left\lfloor\frac{n-k}{2}\right\rfloor,\left[\frac{n-k}{2}\right\rceil}\right)$ for $5 \leq n \leq 11$ and $4 \leq k \leq 10$

\begin{tabular}{lll|lll}
\hline$(n, k)$ & $W\left(C P_{n, k}^{\left[\frac{k}{2}\right\rceil}\right)$ & $W\left(C P_{\left\lfloor\frac{n-k}{2}\right\rfloor,\left[\frac{n-k}{2}\right]}\right)$ & $(n, k)$ & $W\left(C P_{n, k}^{\left[\frac{k}{2}\right]}\right)$ & $W\left(C P_{\left\lfloor\frac{n-k}{2}\right\rfloor,\left[\frac{n-k}{2}\right]}\right)$ \\
\hline$(5,4)$ & 18 & 20 & $(6,4)$ & 28 & 35 \\
\hline$(6,5)$ & 31 & 35 & $(7,4)$ & 40 & 52 \\
\hline$(7,5)$ & 44 & 56 & $(7,6)$ & 50 & 56 \\
\hline$(8,4)$ & 54 & 74 & $(8,5)$ & 59 & 79 \\
\hline$(8,6)$ & 67 & 84 & $(8,7)$ & 75 & 108 \\
\hline$(9,4)$ & 70 & 98 & $(9,5)$ & 76 & 120 \\
\hline$(9,6)$ & 86 & 114 & $(9,7)$ & 96 & 127 \\
\hline$(9,8)$ & 108 & 120 & $(10,4)$ & 88 & 151 \\
\hline$(10,5)$ & 95 & 139 & $(10,6)$ & 107 & 165 \\
\hline$(10,7)$ & 119 & 158 & $(10,8)$ & 134 & 190 \\
\hline$(10,9)$ & 149 & 165 & $(11,4)$ & 108 & 212 \\
\hline$(11,5)$ & 116 & 176 & $(11,6)$ & 130 & 220 \\
\hline$(11,9)$ & 144 & 204 & $(11,8)$ & 162 & \\
\hline
\end{tabular}

\section{References}

[1] D. Vukičević, J. Sedlar, On indices of Wiener and anti-Wiener type, Discrete Appl. Math. 251 (2018) 290-298.

[2] H. Wiener, Structural determination of paraffin boiling points, J. Amer. Chem. Soc. 69 (1947) 17-20.

[3] M. Randić, Novel molecular descriptor for structure-property studies, Chem. Phys. Lett. 211 (1993) 478-483.

[4] M. Randić, Wiener-Hosoya index-A novel graph theoretical molecular descriptor. J. Chem. Inf. Comput. Sci. 44 (2004) 373-377.

[5] D. J. Klein, Z. Mihalić, D. Plavšić and N. Trimajstrić, Molecular topological index, a relation with the Wiener index, J. Chem. Inf. Comput. Sci. 32 (1992) 304-305.

[6] I. Gutman, Selected properties of the Schultz molecular topological index, J. Chem. Inf. Comput. Sci. 34 (1994) 1087-1089.

[7] A. Graovac, M. Ghorbani, A new version of atom-bond connectivity index, Acta Chim. Slov. 57(3)(2010) 609-612.

[8] M. Rostami, M. Sohrabi-Haghighat, Further Results on New Version of Atom-Bond Connectivity Index, MATCH Commun. Math. Comput. Chem. 71(1)(2014) 21-32.

[9] V. Andova, D. Dimitrov, J. Fink, R. Š krekovski, Bounds on Gutman Index, MATCH Commun. Math. Comput. Chem. 67(2) (2012) 515-524.

[10] C. Jordan, Sur les assemblages de lignes, J. Reine Angew. Math. 70 (1869) 185-190.

[11] B. Ma, B. Wu, W. Zhang, Proximity and average eccentricity of a graph, Inf. Process. Lett. 112 (10) (2012) 392-395.

[12] X. Li, Y. Mao, I. Gutman, The Steiner Wiener index of a graph, Discuss. Math. Graph Theory, 36 (2016) 455-465.

[13] L. Lu, Q. Huang, J. Hou and X. Chen, A sharp lower bound on Steiner Wiener index for trees with given diameter, Discrete Math. 341 (2018) 723-731. 
[14] J. Zhang, G. Zhang, H. Wang and X. Zhang, Extremal trees with respect to the Stiener Wiener index, Discrete Math. Algorithms Appl. 11 (6) (2019) 1950067.

[15] I. Gutman, W. Linert, I. Lukovits, A.A. Dobrynin, Trees with extremal hyper-Wiener index: Mathematical basis and chemical applications, J. Chem. Inf. Comput. Sci. 37 (1997) 349-354.

[16] G. Yu, L. Feng, A. Ilić, The hyper-Wiener index of trees with given parameters, Ars Combinatoria, 96(2010) 395-404.

[17] G. Cai, G. Yu, J. Cao, et al., The Hyper-Wiener Index of Trees of Order $n$ with Diameter $d$, Discrete Dyn Nat Soc, 2016.

[18] H. Feng, J. Qian, Minimum trees with respect to Wiener-Hosoya index, Journal of Mathematical Study, 39 (2) (2006) 117-123.

[19] I. Tomescu, Some extremal properties of the degree distance of a graph, Discrete Appl. Math. 98 (1999) 159-163.

[20] D. Bonchev, N. Trinajstić, Information theory, distance matrix, and molecular branching, J. Chem. Phys. 67 (1977) 4517-4533.

[21] H. Liu, X. Pan, On the Wiener index of trees with fixed diameter, MATCH Commun. Math. Comput. Chem. 60 (2008) 85-94.

[22] S. Wang, X. Guo, Trees with extremal Wiener indices, MATCH Commun. Math. Comput. Chem. 60 (2008) 609-622.

[23] D. Pandey, K. L. Patra, Wiener index of graphs with fixed number of pendant or cut vertices, 2019, arXiv:1907.13481v1.

[24] S. Nikolić, N. Trinajstić, M. Randić, Wiener index revisited, Chem. Phys. Lett. 333(3-4)(2001) 319-321.

[25] I. Gutman, D. Vukičević, J. Žerovnik, A class of modified Wiener indices, Croat. Chem. Acta, 77 (1-2) (2004) 103-109.

[26] M. Liu, B. Liu, On the variable Wiener indices of trees with given maximum degree, Math. Comput. Model. 52 (9-10) (2010) 1651-1659.

[27] Y. Chen, On the $\lambda$-modified Wiener index, Journal of Southwest University for Nationalities(Natural science Edition), 35 (2) (2009) 241-243.

[28] B. Zhang, B. Zhou, On Modified and Reverse Wiener Indices of Trees, Zeitschrift für Naturforschung A, 61 (10-11) (2006) 536-540.

[29] B. Zhang, B. Zhou, Modified Wiener indices of trees, MATCH Commun. Math. Comput. Chem. 56 (2006) 179-188.

[30] D. Vukičević, J. Žerovnik, Variable Wiener indices, MATCH Commun. Math. Comput. Chem. 53 (2005) 385-402. 